\title{
Quantum chemical modeling of mechanical properties of aspirin polymorphic modifications
}

Yevhenii Vaksler ${ }^{\mathrm{a}, \mathrm{b}, \mathrm{c}}$, Abdenacer Idrissi ${ }^{\mathrm{a}}$, Victoriya V. Urzhuntseva ${ }^{\mathrm{b}}$, Svitlana V. Shishkina ${ }^{\mathrm{b}, \mathrm{c}}$

${ }^{a}$ Laboratoire de Spectroscopie pour les Interactions, la Réactivité et l'environnement (UMR CNRS A8516), Université de Lille, 59655, Villeneuve d'Ascq Cedex, France.

${ }^{b}$ V.N. Karazin Kharkiv National University, 4 Svobody Sq., Kharkiv, 61022, Ukraine

${ }^{c}$ SSI “Institute for Single Crystals” NAS of Ukraine, 60 Nauky ave., Kharkiv, 61001, Ukraine 


\section{List of contents}

Table S1. Symmetry codes, bonding types, interaction energies of the basic monomeric BU with neighboring ones $\left(\mathrm{E}_{\mathrm{int}}, \mathrm{kcal} / \mathrm{mol}\right)$ and the contribution of these energies to the total interaction energy $(\%)$ in the crystal of aspirin form $\mathbf{I}$

. .3

Table S2. Symmetry codes, bonding types, interaction energies of the basic monomeric BU with neighboring ones $\left(\mathrm{E}_{\mathrm{int}}, \mathrm{kcal} / \mathrm{mol}\right)$ and the contribution of these energies to the total interaction energy $(\%)$ in the crystal of aspirin form II

.. .4

Table S3. Symmetry codes, bonding types, interaction energies of the basic monomeric BUs A and B with neighboring ones $\left(\mathrm{E}_{\mathrm{int}}, \mathrm{kcal} / \mathrm{mol}\right)$ and the contribution of these energies to the total interaction energy $(\%)$ in the crystal of aspirin form IV.

Table S4. Symmetry codes, bonding types, interaction energies of the basic dimeric BU with neighboring ones $\left(\mathrm{E}_{\mathrm{int}}, \mathrm{kcal} / \mathrm{mol}\right)$ and the contribution of these energies to the total interaction energy $(\%)$ in the crystal of aspirin form $\mathbf{I}$

Table S5. Symmetry codes, bonding types, interaction energies of the basic dimeric BU with neighboring ones $\left(\mathrm{E}_{\mathrm{int}}, \mathrm{kcal} / \mathrm{mol}\right)$ and the contribution of these energies to the total interaction energy $(\%)$ in the crystal of aspirin form II.

Table S6. Symmetry codes, bonding types, interaction energies of the basic dimeric BUs AA and BB, based on the corresponding molecules $\mathrm{A}$ and $\mathrm{B}$, with neighboring ones $\left(\mathrm{E}_{\mathrm{int}}, \mathrm{kcal} / \mathrm{mol}\right)$ and the contribution of these energies to the total interaction energy (\%) in the crystal of aspirin form IV

Table S7. Interaction energy differences arising between the terminal positions of the shifts in the different directions due to the edge effect using the fixed part expanded by an initial fragment in each direction of the mobile part displacement

Table S8. Interaction energy differences between the barriers extracted from the shift profiles of the (100) layers in the [010] direction with the step sizes 1/50,1/25,1/20,1/10 of a translation and the energy barrier value appearing, when the step size equals $1 / 100$ of a translation.

Table S9. Minimal interatomic distances occurring for the displacements of the BUs in the different crystallographic directions

Figure S1. Packing of the molecules in the crystals of aspirin polymorphs I (at the top), II (in the middle), IV (at the bottom). The projections in the $a$ (on the right), $b$ (in the middle), $c$ (on the left) crystallographic directions are represented.

Figure S2. Packing of the energy vector diagrams representing the distribution of monomeric BUs in the crystals of aspirin polymorphs I (at the top), II (in the middle), IV (at the bottom). The projections in the $a$ (on the right), $b$ (in the middle), $c$ (on the left) crystallographic directions are represented.

Figure S3. Packing of the energy vector diagrams representing the distribution of dimeric BUs in the crystals of aspirin polymorphs I (at the top), II (in the middle), IV (at the bottom). The projections in the $a$ (on the right), $b$ (in the middle), $c$ (on the left) crystallographic directions are represented.

Figure S4. Interaction energy profile for the shifts of different symmetric equivalents of dimeric BUs along the (100) layers in the [010] direction. Blue and pale blue dots correspond to the shifts of the initial dimeric BUs along the $\mathrm{BSM}_{2}$ with higher (big) or lower (small) coordinate by the [100] direction respectively, olive and green dots are for the shifts of the dimeric BUs rotated by $2{ }_{1}$-screw axes once belonging to the polymorphic forms: I (A); II (B); IV, AA-BU as a basic element (C); IV, BB-BU as a basic element (D) .. 14

Figure S5. Interaction energy deviations occurring between the symmetrically equivalent positions of the dimeric BU as mobile part (its initial disposition is in blue, disposition rotated by $2{ }_{1}$-screw axes once is in olive) during the transitions from the edge to the center beside the neighboring (100) layer between the -5 th 
and 10th positions in the [010] direction for the polymorphic forms: I (A); II (B); IV, AA-BU as a basic element (C); IV, BB-BU as a basic element (D)

Figure S6. Profile of the interaction energy between the dimeric mobile part and the neighboring (100) layer during the translation between the equivalent positions in the [010] direction with the step size of 1/100 (red), 1/50 (olive), 1/25 (blue), 1/20 (purple), 1/10 (orange) of the corresponding distance for the polymorphic forms: I (A); II (B); IV, AA-BU as a basic element (C); IV, BB-BU as a basic element (D).. 16

Figure S7. Profiles of the interaction energy (black) and minimal interatomic distance (blue) between the dimeric mobile part and the neighboring (100) layer in the [010] direction for the polymorphic forms: I (A); II (B); IV, AA-BU as a basic element (C); IV, BB-BU as a basic element (D)

Figure S8. Profiles of the interaction energy (black) and minimal interatomic distance (blue) between the dimeric mobile part and the neighboring (100) layer in the [001] direction for the polymorphic forms: I (A); II (B); IV, AA-BU as a basic element (C); IV, BB-BU as a basic element (D)

Figure S9. Profiles of the interaction energy (black) and minimal interatomic distance (blue) between the dimeric mobile part and the neighboring (100) layer in the [011] direction for the polymorphic forms $\mathbf{I}$ (A) and II (B)

Figure S10. Profiles of the interaction energy (black) and minimal interatomic distance (blue) between the dimeric mobile part and the neighboring (001) layer in the [100] direction for the polymorphic form I (A) and the neighboring (-102) layer in the [201] direction for the polymorphic form II (B) respectively .......... 19

Figure S11. Profiles of the interaction energy (black) and minimal interatomic distance (blue) between the dimeric mobile part and the neighboring (001) layers for the polymorphic forms I (A); IV, AA-BU as a basic element (C); IV, BB-BU as a basic element (D) and the neighboring (-102) layer for the polymorphic form II (B) respectively in the [010] direction

Figure S12. Profiles of the interaction energy (black) and minimal interatomic distance (blue) between the dimeric mobile part and the neighboring (-101) layer in the [010] direction for the polymorphic form IV, AA-BU as a basic element (A) and BB-BU as a basic element (B) 
Table S1. Symmetry codes, bonding types, interaction energies of the basic monomeric BU with neighboring ones $\left(\mathrm{E}_{\mathrm{int}}, \mathrm{kcal} / \mathrm{mol}\right)$ and the contribution of these energies to the total interaction energy $(\%)$ in the crystal of aspirin form $\mathbf{I}$

\begin{tabular}{ccccc}
\hline Dimer & Symmetry operation & $\mathrm{E}_{\text {int }}, \mathrm{kcal} / \mathrm{mol}$ & $\begin{array}{c}\text { Contribution to the total } \\
\text { interaction energy, } \%\end{array}$ & Interaction \\
\hline $\mathrm{d} 1$ & $\mathrm{x}, 1+\mathrm{y}, \mathrm{z}$ & -4.9 & 7.7 & non-specific \\
$\mathrm{d} 2$ & $\mathrm{x},-1+\mathrm{y}, \mathrm{z}$ & -4.9 & 7.7 & non-specific \\
$\mathrm{d} 3$ & $1-\mathrm{x}, 1 / 2+\mathrm{y}, 5 / 2-\mathrm{z}$ & -2.1 & 3.3 & non-specific \\
$\mathrm{d} 4$ & $1-\mathrm{x},-1 / 2+\mathrm{y}, 5 / 2-\mathrm{z}$ & -2.1 & 3.3 & non-specific \\
$\mathrm{d} 5$ & $2-\mathrm{x}, 1 / 2+\mathrm{y}, 3 / 2-\mathrm{z}$ & -0.6 & 0.9 & non-specific \\
$\mathrm{d} 6$ & $2-\mathrm{x},-1 / 2+\mathrm{y}, 3 / 2-\mathrm{z}$ & -0.6 & 0.9 & O-H...O, $1.65 \AA, 174^{\circ}$ \\
$\mathrm{d} 7$ & $1-\mathrm{x},-\mathrm{y}, 2-\mathrm{z}$ & -19.9 & 31.5 & Stacking, C...C, $3.32 \AA$ \\
$\mathrm{d} 8$ & $1-\mathrm{x}, 1-\mathrm{y}, 2-\mathrm{z}$ & -5.5 & 8.7 & non-specific \\
$\mathrm{d} 9$ & $2-\mathrm{x},-\mathrm{y}, 2-\mathrm{z}$ & -3.6 & 5.6 & non-specific \\
$\mathrm{d} 10$ & $2-\mathrm{x}, 1-\mathrm{y}, 2-\mathrm{z}$ & -6.0 & 9.5 & $\mathrm{C}-\mathrm{H} \ldots \pi, 2.71 \AA, 178^{\circ}$ \\
$\mathrm{d} 11$ & $\mathrm{x}, 1 / 2-\mathrm{y}, 1 / 2+\mathrm{z}$ & -3.7 & 5.9 & $\mathrm{C}-\mathrm{H} \ldots \pi, 2.71 \AA, 178^{\circ}$ \\
$\mathrm{d} 12$ & $\mathrm{x}, 1 / 2-\mathrm{y},-1 / 2+\mathrm{z}$ & -3.7 & 5.9 & non-specific \\
$\mathrm{d} 13$ & $\mathrm{x}, 3 / 2-\mathrm{y}, 1 / 2+\mathrm{z}$ & -2.8 & 4.4 & non-specific \\
$\mathrm{d} 14$ & $\mathrm{x}, 3 / 2-\mathrm{y},-1 / 2+\mathrm{z}$ & -2.8 & 4.4 & \\
\hline
\end{tabular}


Table S2. Symmetry codes, bonding types, interaction energies of the basic monomeric BU with neighboring ones $\left(\mathrm{E}_{\mathrm{int}}, \mathrm{kcal} / \mathrm{mol}\right)$ and the contribution of these energies to the total interaction energy $(\%)$ in the crystal of aspirin form II

\begin{tabular}{ccccc}
\hline Dimer & Symmetry operation & E int, kcal/mol & $\begin{array}{c}\text { Contribution to the total } \\
\text { interaction energy, } \%\end{array}$ & Interaction \\
\hline $\mathrm{d} 1$ & $\mathrm{x}, 1+\mathrm{y}, \mathrm{z}$ & -4.9 & 7.6 & non-specific \\
$\mathrm{d} 2$ & $\mathrm{x},-1+\mathrm{y}, \mathrm{z}$ & -4.9 & 7.6 & non-specific \\
$\mathrm{d} 3$ & $-\mathrm{x}, 1 / 2+\mathrm{y}, 1 / 2-\mathrm{z}$ & -4.8 & 7.4 & non-specific \\
$\mathrm{d} 4$ & $-\mathrm{x},-1 / 2+\mathrm{y}, 1 / 2-\mathrm{z}$ & -4.8 & 7.4 & non-specific \\
$\mathrm{d} 5$ & $1-\mathrm{x}, 1 / 2+\mathrm{y}, 3 / 2-\mathrm{z}$ & -2.2 & 3.4 & non-specific \\
$\mathrm{d} 6$ & $1-\mathrm{x},-1 / 2+\mathrm{y}, 3 / 2-\mathrm{z}$ & -2.2 & 3.4 & non-specific \\
$\mathrm{d} 7$ & $-\mathrm{x},-\mathrm{y},-\mathrm{z}$ & -0.6 & 0.9 & non-specific \\
$\mathrm{d} 8$ & $1-\mathrm{x},-\mathrm{y}, 1-\mathrm{z}$ & -21.3 & 33.2 & O-H...O, $1.64 \AA, 176^{\circ}$ \\
$\mathrm{d} 9$ & $1-\mathrm{x}, 1-\mathrm{y}, 1-\mathrm{z}$ & -5.5 & 8.6 & Stacking, C...C, $3.36 \AA$ \\
$\mathrm{d} 10$ & $\mathrm{x}, 1 / 2-\mathrm{y}, 1 / 2+\mathrm{z}$ & -3.8 & 5.9 & $\mathrm{C}-\mathrm{H} \ldots \pi, 2.71 \AA, 176^{\circ}$ \\
$\mathrm{d} 11$ & $\mathrm{x}, 1 / 2-\mathrm{y},-1 / 2+\mathrm{z}$ & -3.8 & 5.9 & $\mathrm{C}-\mathrm{H} . . . \pi, 2.71 \AA, 176^{\circ}$ \\
$\mathrm{d} 12$ & $\mathrm{x}, 3 / 2-\mathrm{y}, 1 / 2+\mathrm{z}$ & -2.8 & 4.3 & non-specific \\
$\mathrm{d} 13$ & $\mathrm{x}, 3 / 2-\mathrm{y},-1 / 2+\mathrm{z}$ & -2.8 & 4.3 & non-specific \\
\hline
\end{tabular}


Table S3. Symmetry codes, bonding types, interaction energies of the basic monomeric BUs A and B with neighboring ones $\left(\mathrm{E}_{\mathrm{int}}, \mathrm{kcal} / \mathrm{mol}\right)$ and the contribution of these energies to the total interaction energy $(\%)$ in the crystal of aspirin form IV

\begin{tabular}{|c|c|c|c|c|c|}
\hline Dimer & Symmetry operation & $\mathrm{E}_{\text {int }}, \mathrm{kcal} / \mathrm{mol}$ & $\begin{array}{l}\text { Contribution to the total } \\
\text { interaction energy, } \%\end{array}$ & Interaction & Neighbor type \\
\hline \multicolumn{6}{|c|}{ Monomers neighboring to molecule A } \\
\hline $\mathrm{d} 1$ & $2-x, 2-y, 2-z$ & -2.7 & 2.2 & non-specific & A \\
\hline $\mathrm{d} 2$ & $2-x, 3-y, 2-z$ & -23.2 & 19.1 & $\mathrm{O}-\mathrm{H} \ldots \mathrm{O}, 1.58 \AA, 171^{\circ}$ & A \\
\hline d3 & $1-\mathrm{x}, 1 / 2+\mathrm{y}, 3 / 2-\mathrm{z}$ & -2.1 & 1.7 & $\mathrm{C}-\mathrm{H} \ldots \pi, 2.84 \AA, 140^{\circ}$ & B \\
\hline $\mathrm{d} 4$ & $1-x, 3 / 2+y, 3 / 2-z$ & -1.1 & 0.9 & H...H, $2.11 \AA$ & B \\
\hline d5 & $2-x, 1 / 2+y, 3 / 2-z$ & -4.8 & 4.0 & $\mathrm{C}-\mathrm{H} \ldots \pi, 2.66 \AA, 162^{\circ}$ & A \\
\hline d6 & $2-x, 1 / 2+y, 3 / 2-z$ & -1.8 & 1.5 & non-specific & B \\
\hline d7 & $2-x,-1 / 2+y, 3 / 2-z$ & -4.8 & 4.0 & $\mathrm{C}-\mathrm{H} . . . \pi, 2.66 \AA, 162^{\circ}$ & A \\
\hline $\mathrm{d} 8$ & $1+\mathrm{x}, 1 / 2-\mathrm{y}, 1 / 2+\mathrm{z}$ & -1.4 & 1.1 & non-specific & B \\
\hline d9 & $1+\mathrm{x}, 3 / 2-\mathrm{y}, 1 / 2+\mathrm{z}$ & -2.2 & 1.8 & non-specific & B \\
\hline $\mathrm{d} 10$ & $\mathrm{x}, \mathrm{y}, \mathrm{z}$ & -0.9 & 0.7 & H...H, $2.28 \AA$ & B \\
\hline $\mathrm{d} 11$ & $\mathrm{x}, 1+\mathrm{y}, \mathrm{z}$ & -7.5 & 6.1 & non-specific & A \\
\hline $\mathrm{d} 12$ & $\mathrm{x}, 1+\mathrm{y}, \mathrm{z}$ & -2.3 & 1.9 & non-specific & B \\
\hline $\mathrm{d} 13$ & $\mathrm{x},-1+\mathrm{y}, \mathrm{z}$ & -7.5 & 6.1 & non-specific & A \\
\hline \multicolumn{6}{|c|}{ Monomers neighboring to molecule $\mathrm{B}$} \\
\hline $\mathrm{d} 14$ & $1-x,-y, 1-z$ & -21.0 & 17.3 & O-H...O, $1.67 \AA, 170^{\circ}$ & B \\
\hline d15 & $1-x, 1-y, 1-z$ & -1.9 & 1.6 & non-specific & B \\
\hline d16 & $1-\mathrm{x}, 1 / 2+\mathrm{y}, 3 / 2-\mathrm{z}$ & -5.1 & 4.2 & C-H...O, $2.46 \AA, 130^{\circ}$ & B \\
\hline $\mathrm{d} 17$ & $1-x,-1 / 2+y, 3 / 2-z$ & -5.1 & 4.2 & C-H...O, $2.46 \AA, 130^{\circ}$ & B \\
\hline $\mathrm{d} 18$ & $1-\mathrm{x},-1 / 2+\mathrm{y}, 3 / 2-\mathrm{z}$ & -2.1 & 1.7 & C...H, $2.84 \AA$ & A \\
\hline d19 & $1-x,-3 / 2+y, 3 / 2-z$ & -1.1 & 0.9 & H...H, $2.11 \AA$ & A \\
\hline $\mathrm{d} 20$ & $2-x,-1 / 2+y, 3 / 2-z$ & -1.8 & 1.5 & non-specific & A \\
\hline $\mathrm{d} 21$ & $-1+\mathrm{x}, 1 / 2-\mathrm{y},-1 / 2+\mathrm{z}$ & -1.4 & 1.1 & non-specific & A \\
\hline $\mathrm{d} 22$ & $-1+\mathrm{x}, 3 / 2-\mathrm{y},-1 / 2+\mathrm{z}$ & -2.2 & 1.8 & non-specific & A \\
\hline $\mathrm{d} 23$ & $\mathrm{x}, \mathrm{y}, \mathrm{z}$ & -0.9 & 0.7 & H...H, $2.28 \AA$ & A \\
\hline $\mathrm{d} 24$ & $\mathrm{x}, 1+\mathrm{y}, \mathrm{z}$ & -7.3 & 6.0 & Stacking, C...C, $3.36 \AA$ & B \\
\hline $\mathrm{d} 25$ & $\mathrm{x},-1+\mathrm{y}, \mathrm{z}$ & -7.3 & 6.0 & 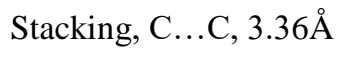 & B \\
\hline $\mathrm{d} 26$ & $\mathrm{x},-1+\mathrm{y}, \mathrm{z}$ & -2.3 & 1.9 & non-specific & A \\
\hline
\end{tabular}


Table S4. Symmetry codes, bonding types, interaction energies of the basic dimeric BU with neighboring ones $\left(\mathrm{E}_{\mathrm{int}}, \mathrm{kcal} / \mathrm{mol}\right)$ and the contribution of these energies to the total interaction energy $(\%)$ in the crystal of aspirin form $\mathbf{I}$

\begin{tabular}{|c|c|c|c|c|}
\hline $\begin{array}{l}\text { Dimer of } \\
\text { dimers }\end{array}$ & Symmetry operation & $\mathrm{E}_{\text {int }}, \mathrm{kcal} / \mathrm{mol}$ & $\begin{array}{l}\text { Contribution to the total } \\
\text { interaction energy, } \%\end{array}$ & Interaction \\
\hline $\mathrm{dd} 1$ & $\mathrm{x}, 1+\mathrm{y}, \mathrm{z}$ & -13.4 & 17.8 & Stacking, C...C, $3.32 \AA$ \\
\hline $\mathrm{dd} 2$ & $\mathrm{x},-1+\mathrm{y}, \mathrm{z}$ & -13.4 & 17.8 & Stacking, C...C, $3.32 \AA$ \\
\hline $\mathrm{dd} 3$ & $1+\mathrm{x}, \mathrm{y}, \mathrm{z}$ & -1.6 & 2.1 & non-specific \\
\hline $\mathrm{dd} 4$ & $1+\mathrm{x}, 1+\mathrm{y}, \mathrm{z}$ & -4.6 & 6.0 & non-specific \\
\hline dd5 & $-1+\mathrm{x}, \mathrm{y}, \mathrm{z}$ & -1.6 & 2.1 & non-specific \\
\hline dd6 & $-1+\mathrm{x},-1+\mathrm{y}, \mathrm{z}$ & -4.6 & 6.0 & non-specific \\
\hline $\mathrm{dd} 7$ & $-x, 1 / 2+y, 5 / 2-z$ & -0.8 & 1.0 & non-specific \\
\hline $\mathrm{dd} 8$ & $-x,-1 / 2+y, 5 / 2-z$ & -0.8 & 1.0 & non-specific \\
\hline dd9 & $1-\mathrm{x}, 1 / 2+\mathrm{y}, 3 / 2-\mathrm{z}$ & -5.5 & 7.3 & $\mathrm{C}-\mathrm{H} \ldots \pi, 2.71 \AA \AA^{\circ}, 178^{\circ}$ \\
\hline $\mathrm{dd} 10$ & $1-x, 1 / 2+y, 5 / 2-z$ & -5.5 & 7.3 & C-H... $2.71 \AA, 178^{\circ}$ \\
\hline dd11 & $1-x, 3 / 2+y, 3 / 2-z$ & -2.8 & 3.7 & non-specific \\
\hline $\mathrm{dd} 12$ & $1-x, 3 / 2+y, 5 / 2-z$ & -2.8 & 3.7 & non-specific \\
\hline $\mathrm{dd} 13$ & $1-\mathrm{x},-1 / 2+\mathrm{y}, 3 / 2-\mathrm{z}$ & -5.5 & 7.3 & $\mathrm{C}-\mathrm{H} \ldots \pi, 2.71 \AA, 178^{\circ}$ \\
\hline dd14 & $1-x,-1 / 2+y, 5 / 2-z$ & -5.5 & 7.3 & C-H... $\pi, 2.71 \AA, 178^{\circ}$ \\
\hline dd 15 & $1-x,-3 / 2+y, 3 / 2-z$ & -2.8 & 3.7 & non-specific \\
\hline $\operatorname{dd} 16$ & $1-x,-3 / 2+y, 5 / 2-z$ & -2.8 & 3.7 & non-specific \\
\hline $\operatorname{dd} 17$ & $2-x, 1 / 2+y, 3 / 2-z$ & -0.8 & 1.0 & non-specific \\
\hline $\operatorname{dd} 18$ & $2-x,-1 / 2+y, 3 / 2-z$ & -0.8 & 1.0 & non-specific \\
\hline
\end{tabular}


Table S5. Symmetry codes, bonding types, interaction energies of the basic dimeric BU with neighboring ones $\left(\mathrm{E}_{\mathrm{int}}, \mathrm{kcal} / \mathrm{mol}\right)$ and the contribution of these energies to the total interaction energy $(\%)$ in the crystal of aspirin form II

\begin{tabular}{|c|c|c|c|c|}
\hline $\begin{array}{l}\text { Dimer of } \\
\text { dimers }\end{array}$ & Symmetry operation & $\mathrm{E}_{\mathrm{int}}, \mathrm{kcal} / \mathrm{mol}$ & $\begin{array}{l}\text { Contribution to the total } \\
\text { interaction energy, } \%\end{array}$ & Interaction \\
\hline $\mathrm{dd} 1$ & $\mathrm{x}, 1+\mathrm{y}, \mathrm{z}$ & -13.4 & 17.8 & Stacking, C...C, $3.36 \AA$ \\
\hline $\mathrm{dd} 2$ & $\mathrm{x},-1+\mathrm{y}, \mathrm{z}$ & -13.4 & 17.8 & Stacking, C...C, $3.36 \AA$ \\
\hline $\mathrm{dd} 3$ & $1+\mathrm{x}, \mathrm{y}, 1+\mathrm{z}$ & -0.8 & 1.0 & non-specific \\
\hline dd 4 & $-1+\mathrm{x}, \mathrm{y},-1+\mathrm{z}$ & -0.8 & 1.0 & non-specific \\
\hline dd5 & $-\mathrm{x}, 1 / 2+\mathrm{y}, 1 / 2-\mathrm{z}$ & -3.3 & 4.4 & non-specific \\
\hline dd 6 & $-\mathrm{x},-1 / 2+\mathrm{y}, 1 / 2-\mathrm{z}$ & -3.3 & 4.4 & non-specific \\
\hline $\mathrm{dd} 7$ & $1-\mathrm{x}, 1 / 2+\mathrm{y}, 1 / 2-\mathrm{z}$ & -5.6 & 7.4 & $\mathrm{C}-\mathrm{H} \ldots \pi, 2.71 \AA, 176^{\circ}$ \\
\hline $\mathrm{dd} 8$ & $1-\mathrm{x}, 1 / 2+\mathrm{y}, 3 / 2-\mathrm{z}$ & -5.6 & 7.4 & $\mathrm{C}-\mathrm{H} \ldots \pi, 2.71 \AA, 176^{\circ}$ \\
\hline dd 9 & $1-\mathrm{x}, 3 / 2+\mathrm{y}, 1 / 2-\mathrm{z}$ & -2.8 & 3.7 & non-specific \\
\hline $\mathrm{dd} 10$ & $1-x, 3 / 2+y, 3 / 2-z$ & -2.8 & 3.7 & non-specific \\
\hline $\mathrm{dd} 11$ & $1-\mathrm{x},-1 / 2+\mathrm{y}, 1 / 2-\mathrm{z}$ & -5.6 & 7.4 & C-H... $2.71 \AA$ A, $176^{\circ}$ \\
\hline $\mathrm{dd} 12$ & $1-x,-1 / 2+y, 3 / 2-z$ & -5.6 & 7.4 & $\mathrm{C}-\mathrm{H} \ldots \pi, 2.71 \AA, 176^{\circ}$ \\
\hline $\mathrm{dd} 13$ & $1-x,-3 / 2+y, 1 / 2-z$ & -2.8 & 3.7 & non-specific \\
\hline dd14 & $1-x,-3 / 2+y, 3 / 2-z$ & -2.8 & 3.7 & non-specific \\
\hline dd15 & $2-\mathrm{x}, 1 / 2+\mathrm{y}, 3 / 2-\mathrm{z}$ & -3.3 & 4.4 & non-specific \\
\hline $\operatorname{dd} 16$ & $2-x,-1 / 2+y, 3 / 2-z$ & -3.3 & 4.4 & non-specific \\
\hline
\end{tabular}


Table S6. Symmetry codes, bonding types, interaction energies of the basic dimeric BUs AA and BB, based on the corresponding molecules $\mathrm{A}$ and $\mathrm{B}$, with neighboring ones $\left(\mathrm{E}_{\mathrm{int}}, \mathrm{kcal} / \mathrm{mol}\right)$ and the contribution of these energies to the total interaction energy (\%) in the crystal of aspirin form IV

\begin{tabular}{|c|c|c|c|c|c|}
\hline Dimer & Symmetry operation & $\mathrm{E}_{\text {int }}, \mathrm{kcal} / \mathrm{mol}$ & $\begin{array}{l}\text { Contribution to the total } \\
\text { interaction energy, \% }\end{array}$ & Interaction & Neighbor type \\
\hline \multicolumn{6}{|c|}{ Dimers neighboring to AA-BU } \\
\hline $\mathrm{dd} 1$ & $\mathrm{x},-1+\mathrm{y}, \mathrm{z}$ & -17.7 & 11.2 & non-specific & $\mathrm{AA}$ \\
\hline $\mathrm{dd} 2$ & $\mathrm{x}, 1+\mathrm{y}, \mathrm{z}$ & -17.7 & 11.2 & non-specific & AA \\
\hline $\mathrm{dd} 3$ & $2-x,-0.5+y, 1.5-z$ & -4.9 & 3.1 & $\mathrm{C}-\mathrm{H} \ldots \pi, 2.68 \AA, 163^{\circ}$ & AA \\
\hline $\mathrm{dd} 4$ & $2-x,-0.5+y, 2.5-z$ & -4.9 & 3.1 & $\mathrm{C}-\mathrm{H} \ldots \pi, 2.68 \AA, 163^{\circ}$ & AA \\
\hline dd5 & $2-x, 0.5+y, 1.5-z$ & -4.9 & 3.1 & $\mathrm{C}-\mathrm{H} \ldots \pi, 2.68 \AA, 163^{\circ}$ & AA \\
\hline dd6 & $2-x, 0.5+y, 2.5-z$ & -4.9 & 3.1 & $\mathrm{C}-\mathrm{H} \ldots \pi, 2.68 \AA, 163^{\circ}$ & $\mathrm{AA}$ \\
\hline $\mathrm{dd} 7$ & $1-x, 0.5+y, 1.5-z$ & -2.2 & 1.4 & non-specific & BB \\
\hline $\mathrm{dd} 8$ & $1-\mathrm{x}, 1.5+\mathrm{y}, 1.5-\mathrm{z}$ & -3.0 & 1.9 & non-specific & BB \\
\hline dd 9 & $1-\mathrm{x}, 2.5+\mathrm{y}, 1.5-\mathrm{z}$ & -3.6 & 2.3 & non-specific & BB \\
\hline dd10 & $\mathrm{x}, \mathrm{y}, \mathrm{z}$ & -1.2 & 0.8 & non-specific & BB \\
\hline dd11 & $\mathrm{x}, 1+\mathrm{y}, \mathrm{z}$ & -2.4 & 1.5 & non-specific & BB \\
\hline $\mathrm{dd} 12$ & $2-x, 0.5+y, 1.5-z$ & -3.6 & 2.3 & non-specific & BB \\
\hline $\operatorname{dd} 13$ & $2-x, 1.5+y, 1.5-z$ & -2.9 & 1.9 & non-specific & BB \\
\hline $\operatorname{dd} 14$ & $2-x, 2.5+y, 1.5-z$ & -2.2 & 1.4 & non-specific & BB \\
\hline dd15 & $1+\mathrm{x}, 2+\mathrm{y}, 1+\mathrm{z}$ & -2.4 & 1.5 & non-specific & BB \\
\hline $\operatorname{dd} 16$ & $1+\mathrm{x}, 3+\mathrm{y}, 1+\mathrm{z}$ & -1.2 & 0.8 & non-specific & BB \\
\hline \multicolumn{6}{|c|}{ Dimers neighboring to $\mathrm{BB}-\mathrm{BU}$} \\
\hline dd17 & $\mathrm{x}, 1+\mathrm{y}, \mathrm{z}$ & -16.5 & 10.4 & Stacking, C...C, $3.36 \AA$ & BB \\
\hline $\mathrm{dd} 18$ & $\mathrm{x},-1+\mathrm{y}, \mathrm{z}$ & -16.5 & 10.4 & Stacking, C...C, $3.36 \AA$ & BB \\
\hline dd19 & $1-x, 0.5+y, 0.5-z$ & -5.2 & 3.3 & non-specific & BB \\
\hline $\mathrm{dd} 20$ & $1-x,-0.5+y, 0.5-z$ & -5.2 & 3.3 & non-specific & BB \\
\hline $\mathrm{dd} 21$ & $1-x, 0.5+y, 1.5-z$ & -5.2 & 3.3 & non-specific & BB \\
\hline $\operatorname{dd} 22$ & $1-\mathrm{x},-0.5+\mathrm{y}, 1.5-\mathrm{z}$ & -5.2 & 3.3 & non-specific & BB \\
\hline $\operatorname{dd} 23$ & $2-x,-0.5+y, 1.5-z$ & -3.6 & 2.3 & non-specific & AA \\
\hline $\operatorname{dd} 24$ & $2-x,-1.5+y, 1.5-z$ & -2.9 & 1.9 & non-specific & AA \\
\hline $\operatorname{dd} 25$ & $2-x,-2.5+y, 1.5-z$ & -2.2 & 1.4 & non-specific & AA \\
\hline $\mathrm{dd} 26$ & $1-x,-0.5+y, 1.5-z$ & -2.2 & 1.4 & non-specific & AA \\
\hline $\operatorname{dd} 27$ & $1-x,-1.5+y, 1.5-z$ & -2.9 & 1.9 & non-specific & AA \\
\hline $\mathrm{dd} 28$ & $1-x,-2.5+y, 1.5-z$ & -3.6 & 2.3 & non-specific & AA \\
\hline $\mathrm{dd} 29$ & $-1+\mathrm{x},-2+\mathrm{y},-1+\mathrm{z}$ & -2.4 & 1.5 & non-specific & AA \\
\hline $\operatorname{dd} 30$ & $-1+x,-3+y,-1+z$ & -1.2 & 0.8 & non-specific & AA \\
\hline $\mathrm{dd} 31$ & $\mathrm{x},-1+\mathrm{y}, \mathrm{z}$ & -2.4 & 1.5 & non-specific & AA \\
\hline $\mathrm{dd} 32$ & $\mathrm{x}, \mathrm{y}, \mathrm{z}$ & -1.2 & 0.8 & non-specific & AA \\
\hline
\end{tabular}


Table S7. Interaction energy differences arising between the terminal positions of the shifts in the different directions due to the edge effect using the fixed part expanded by an initial fragment in each direction of the mobile part displacement

\begin{tabular}{|c|c|c|c|c|}
\hline \multirow{2}{*}{ Shift direction } & \multicolumn{4}{|c|}{$\Delta \mathrm{E}_{\mathrm{int}}, \mathrm{kcal} / \mathrm{mol}$} \\
\hline & I & II & IV, AA-basic element & IV, BB-basic element \\
\hline$\left[\begin{array}{lll}1 & 0 & 0\end{array}\right]\left(\begin{array}{lll}0 & 0 & 1\end{array}\right)$ & 0.0 & - & - & - \\
\hline$\left[\begin{array}{lll}2 & 0 & 1\end{array}\right]\left(\begin{array}{lll}-1 & 0 & 2\end{array}\right)$ & - & 0.0 & - & - \\
\hline$\left[\begin{array}{lll}0 & 1 & 0\end{array}\right]\left(\begin{array}{lll}-1 & 0 & 1\end{array}\right)$ & - & - & 0.1 & 0.0 \\
\hline$\left[\begin{array}{lll}0 & 1 & 0\end{array}\right]\left(\begin{array}{lll}0 & 0 & 1\end{array}\right)$ & 0.2 & 0.1 & 0.1 & 0.0 \\
\hline$\left[\begin{array}{lll}0 & 1 & 0\end{array}\right]\left(\begin{array}{lll}1 & 0 & 0\end{array}\right)$ & 0.0 & 0.0 & 0.1 & 0.0 \\
\hline$\left[\begin{array}{lll}0 & 0 & 1\end{array}\right]\left(\begin{array}{lll}1 & 0 & 0\end{array}\right)$ & 0.0 & 0.0 & 0.0 & 0.0 \\
\hline$\left[\begin{array}{llll}0 & 1 & 1\end{array}\right]\left(\begin{array}{lll}1 & 0 & 0\end{array}\right)$ & 0.0 & 0.0 & - & - \\
\hline
\end{tabular}

Table S8. Interaction energy differences between the barriers extracted from the shift profiles of the (100) layers in the [010] direction with the step sizes 1/50,1/25, 1/20, 1/10 of a translation and the energy barrier value appearing, when the step size equals $1 / 100$ of a translation

\begin{tabular}{ccccc}
\hline $\begin{array}{c}\text { Step size (in parts of } \\
\text { translation) }\end{array}$ & I & II & IV, AA-basic element & IV, BB-basic element \\
\cline { 2 - 4 } $1 / 50$ & 0.0 & 0.0 & 0.0 & 0.0 \\
$1 / 25$ & 0.0 & 0.0 & 0.0 & 0.0 \\
$1 / 20$ & 0.0 & 0.1 & 0.0 & 0.0 \\
$1 / 10$ & 0.0 & 0.1 & 0.2 & 0.2 \\
\hline
\end{tabular}


Table S9. Minimal interatomic distances occurring for the displacements of the BUs in the different crystallographic directions

\begin{tabular}{|c|c|c|c|c|}
\hline \multirow{2}{*}{ Shift direction } & \multicolumn{4}{|c|}{ Minimal interatomic distance, $\AA$} \\
\hline & $\mathbf{I}$ & II & IV, AA-basic element & IV, BB-basic element \\
\hline$\left[\begin{array}{lll}0 & 1 & 0\end{array}\right]\left(\begin{array}{lll}1 & 0 & 0\end{array}\right)$ & 1.61 & 1.69 & 1.73 & 1.73 \\
\hline$\left[\begin{array}{lll}0 & 0 & 1\end{array}\right]\left(\begin{array}{lll}1 & 0 & 0\end{array}\right)$ & 1.19 & 1.26 & 1.28 & 1.28 \\
\hline$\left[\begin{array}{lll}0 & 1 & 1\end{array}\right]\left(\begin{array}{lll}1 & 0 & 0\end{array}\right)$ & 1.46 & 1.30 & $0.86^{*}$ & $0.77 *$ \\
\hline$\left[\begin{array}{lll}1 & 0 & 0\end{array}\right]\left(\begin{array}{lll}0 & 0 & 1\end{array}\right)$ & 1.15 & - & $0.37 *$ & $0.37 *$ \\
\hline$\left[\begin{array}{lll}0 & 1 & 0\end{array}\right]\left(\begin{array}{lll}0 & 0 & 1\end{array}\right)$ & 1.41 & - & 1.73 & 1.73 \\
\hline$\left[\begin{array}{lll}1 & 1 & 0\end{array}\right]\left(\begin{array}{lll}0 & 0 & 1\end{array}\right)$ & $0.44^{*}$ & - & $0.32 *$ & $0.87 *$ \\
\hline$\left[\begin{array}{lll}0 & 1 & 0\end{array}\right]\left(\begin{array}{lll}-1 & 0 & 2\end{array}\right)$ & - & 1.41 & - & - \\
\hline$\left[\begin{array}{llll}2 & 0 & 1\end{array}\right]\left(\begin{array}{lll}-1 & 0 & 2\end{array}\right)$ & - & 1.07 & - & - \\
\hline$\left[\begin{array}{lll}2 & 1 & 1\end{array}\right]\left(\begin{array}{lll}-1 & 0 & 2\end{array}\right)$ & - & $0.62 *$ & - & - \\
\hline$\left[\begin{array}{lll}0 & 1 & 0\end{array}\right]\left(\begin{array}{lll}-1 & 0 & 1\end{array}\right)$ & - & - & 1.82 & 1.82 \\
\hline$\left[\begin{array}{lll}1 & 0 & 1\end{array}\right]\left(\begin{array}{lll}-1 & 0 & 1\end{array}\right)$ & - & - & $0.32 *$ & $0.17 *$ \\
\hline$\left[\begin{array}{lll}1 & 1 & 1\end{array}\right]\left(\begin{array}{lll}-1 & 0 & 1\end{array}\right)$ & - & - & $>0.01 * *$ & $0.14^{*}$ \\
\hline
\end{tabular}

* Displacements excluded from calculations due to the considerable shortening of the interatomic distances ** Approaching of the mobile and fixed parts closer than $0.01 \AA$ A has been set as the computational limit 


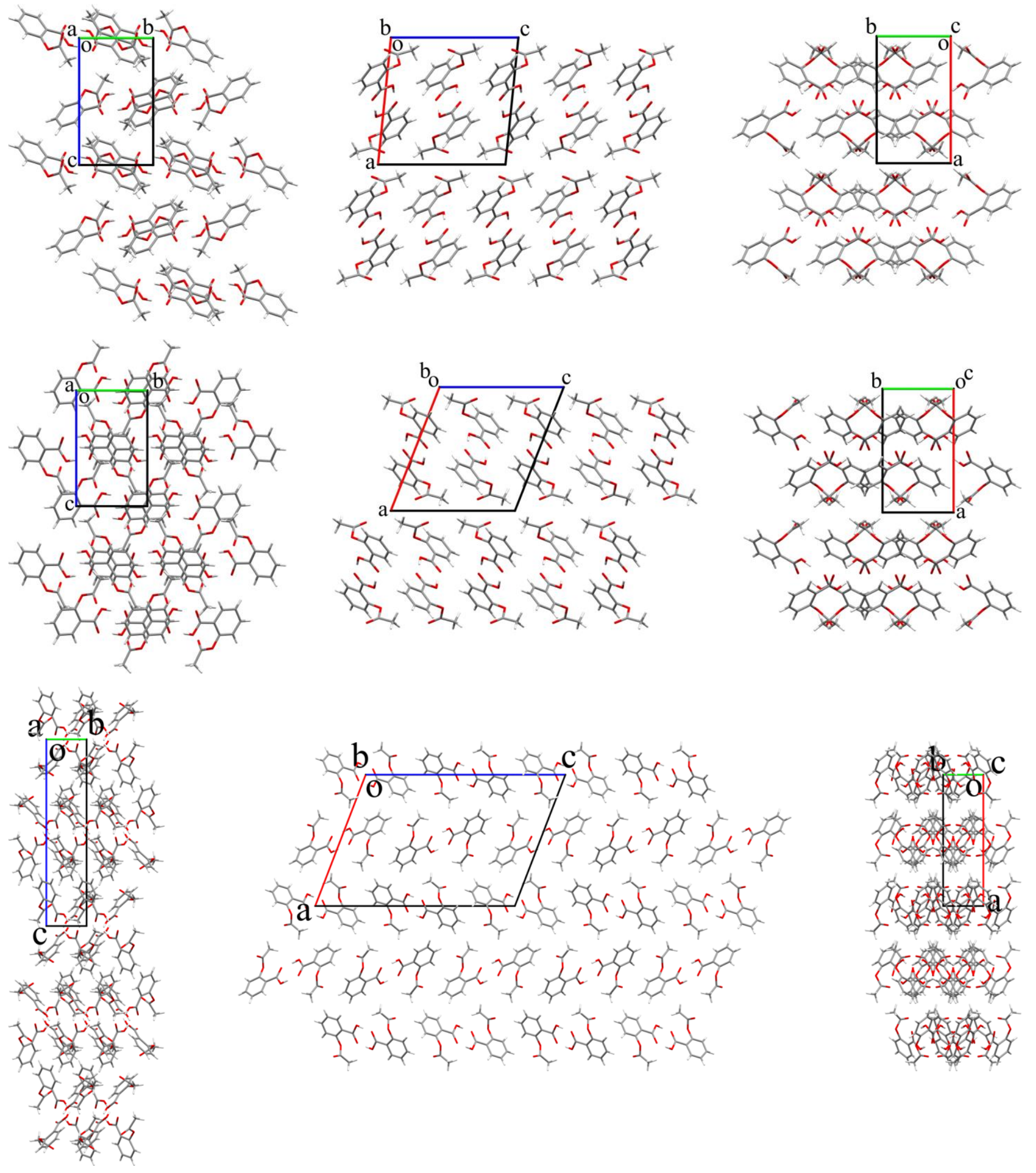

Figure S1. Packing of the molecules in the crystals of aspirin polymorphs I (at the top), II (in the middle), IV (at the bottom). The projections in the $a$ (on the right), $b$ (in the middle), $c$ (on the left) crystallographic directions are represented. 

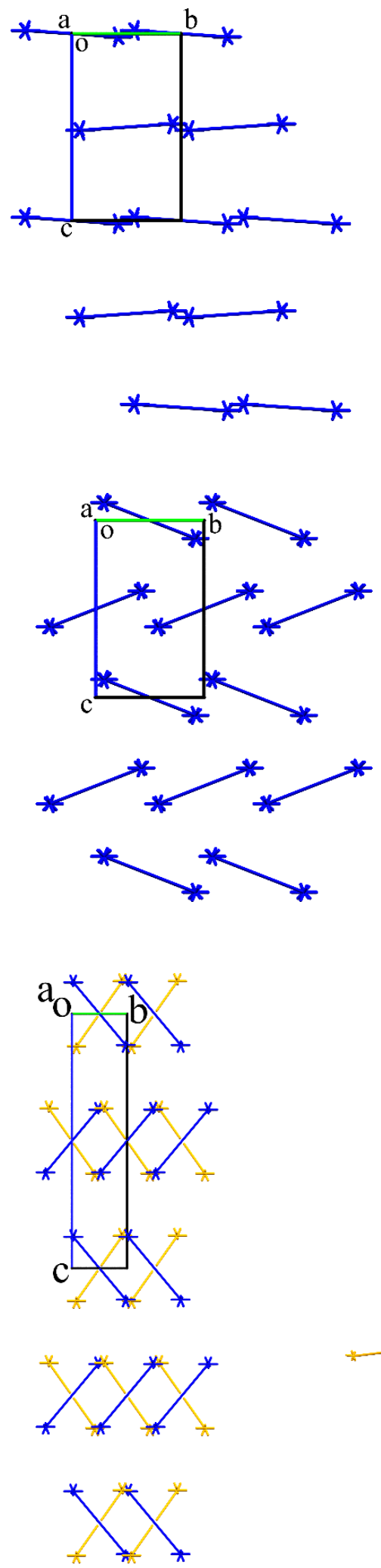
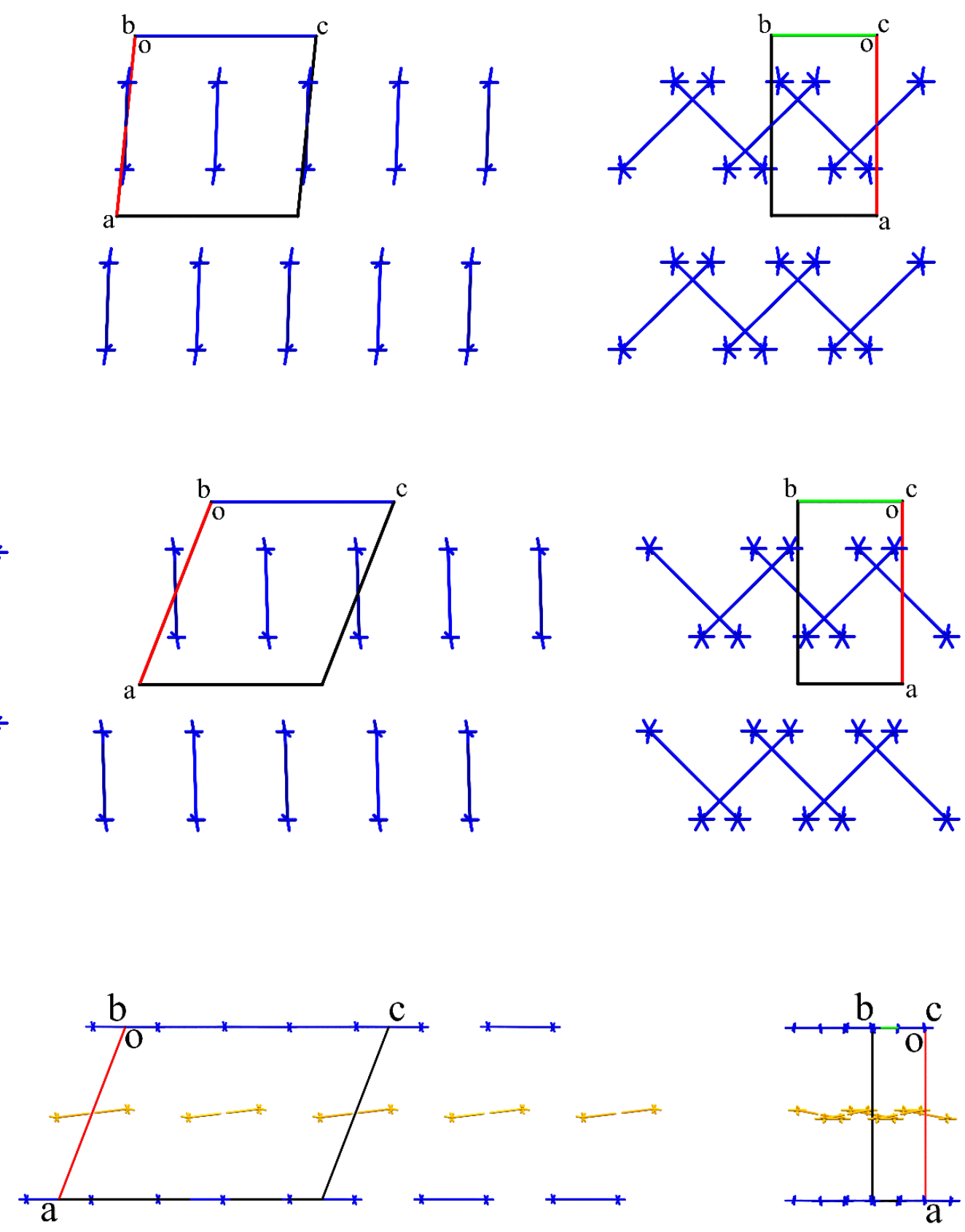

$* * * * *$

Figure S2. Packing of the energy vector diagrams representing the distribution of monomeric BUs in the crystals of aspirin polymorphs I (at the top), II (in the middle), IV (at the bottom). The projections in the $a$ (on the right), $b$ (in the middle), $c$ (on the left) crystallographic directions are represented. 

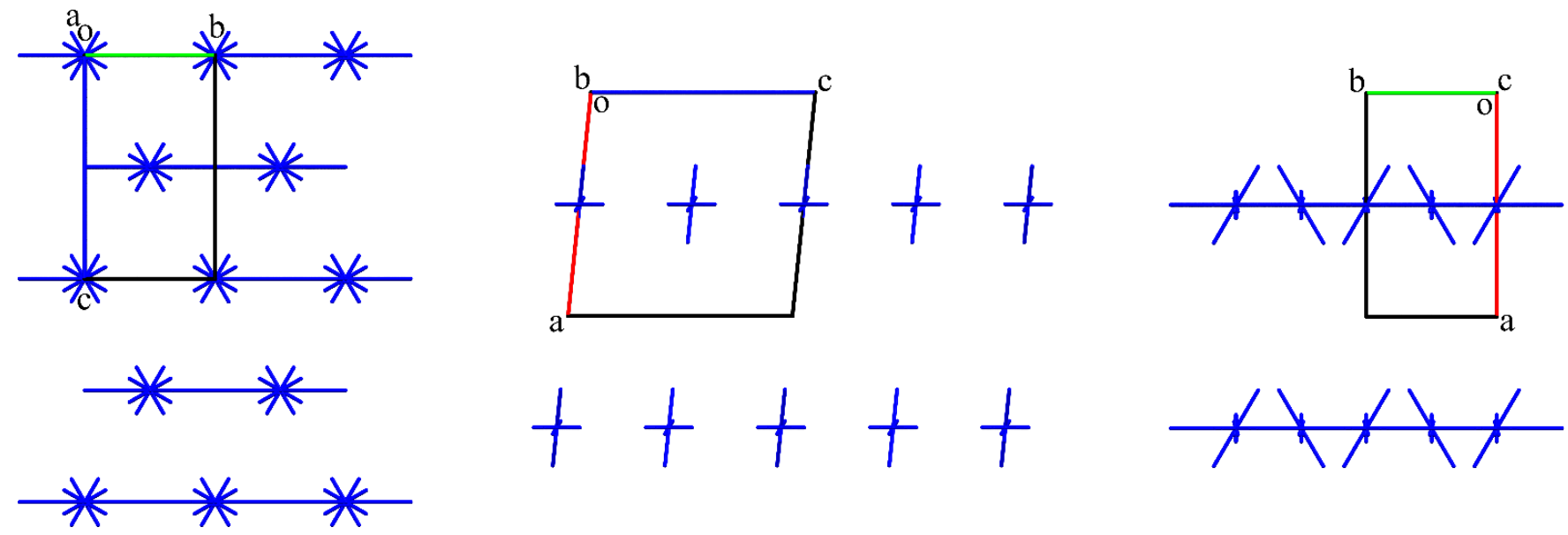

$1+1+1$
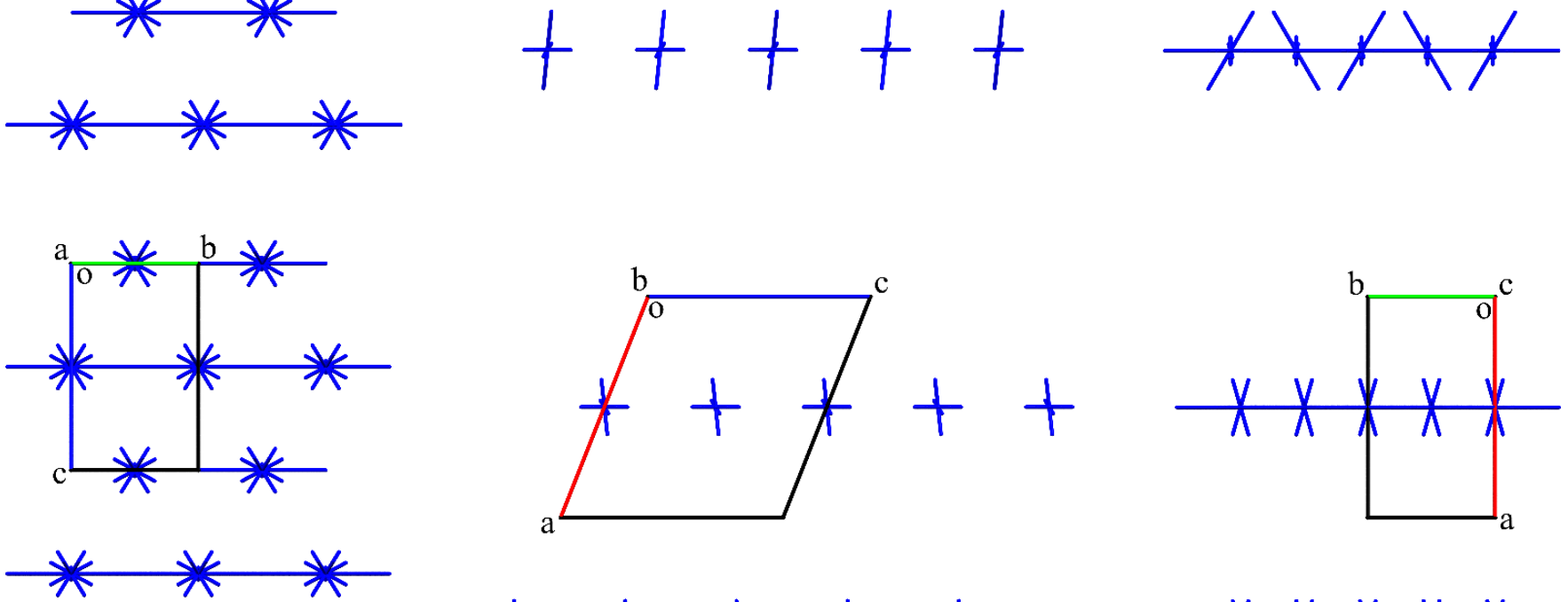

$+1+1+1$
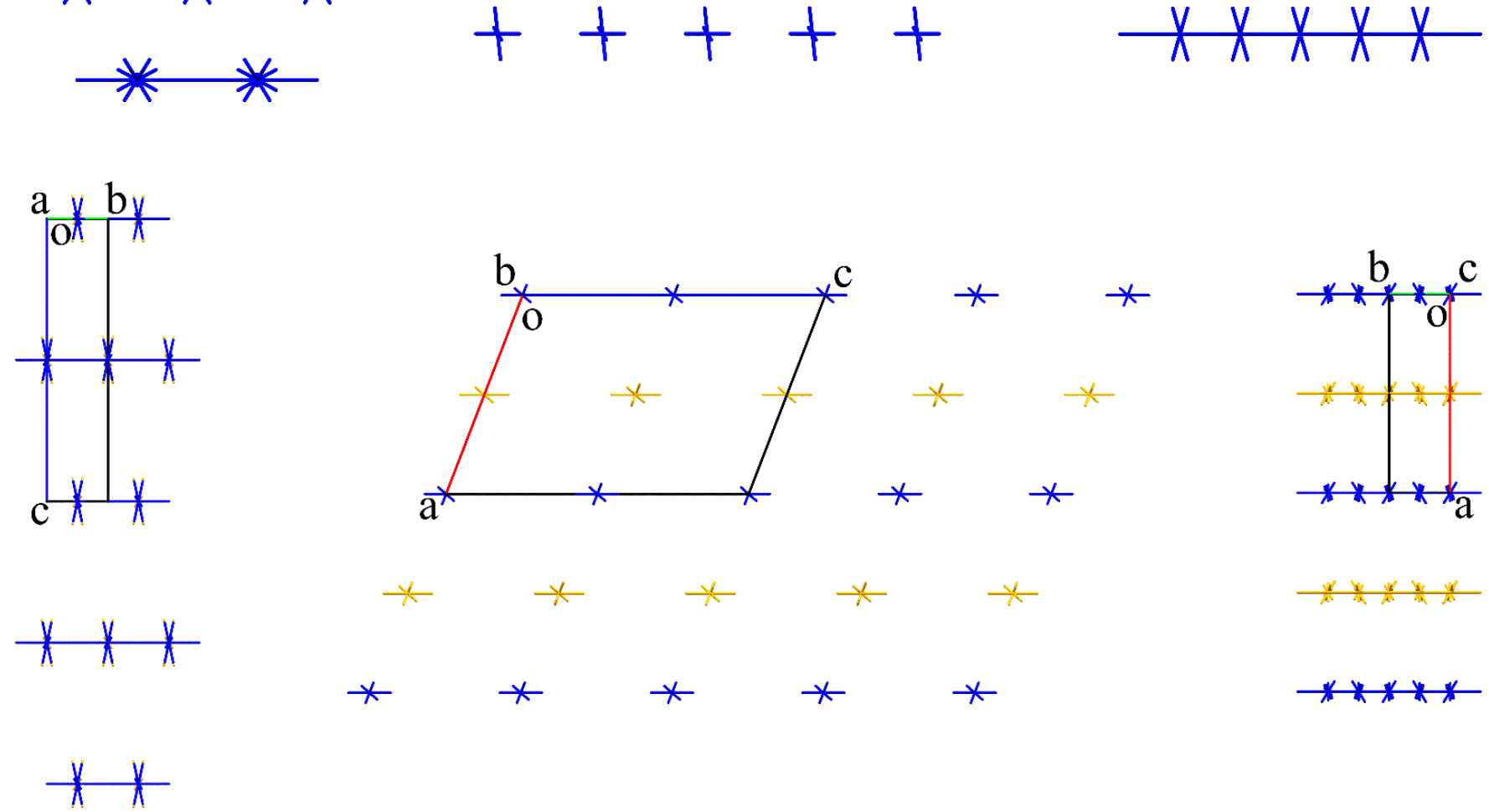

Figure S3. Packing of the energy vector diagrams representing the distribution of dimeric BUs in the crystals of aspirin polymorphs I (at the top), II (in the middle), IV (at the bottom). The projections in the $a$ (on the right), $b$ (in the middle), $c$ (on the left) crystallographic directions are represented. 


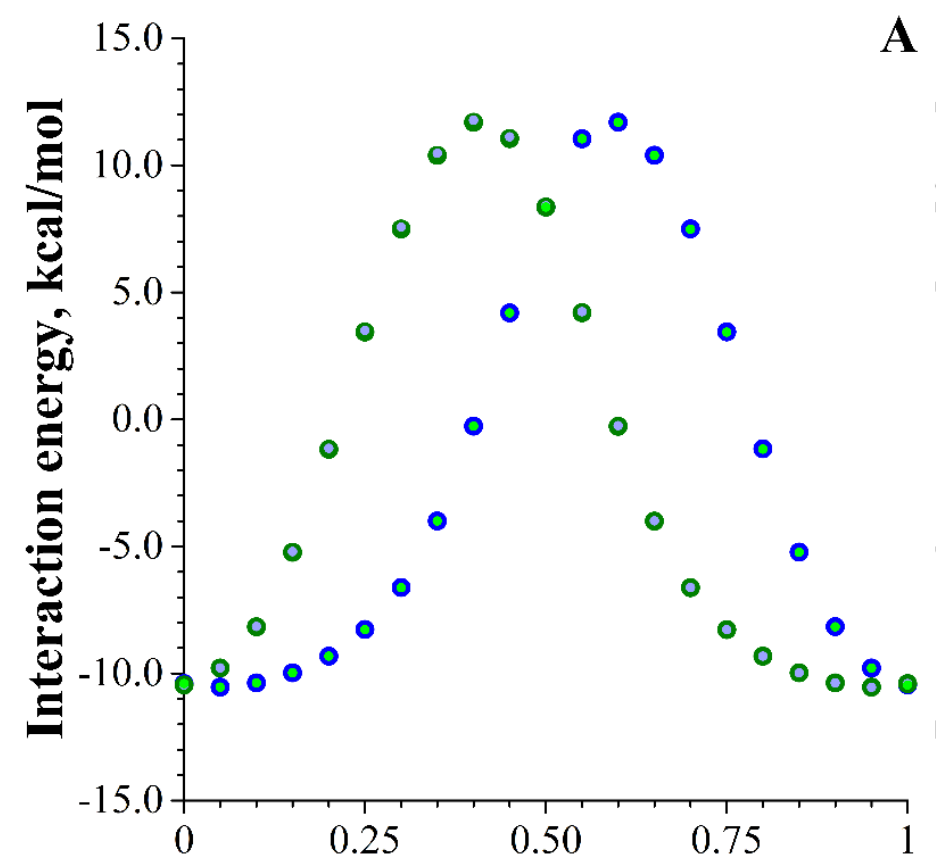

Part of translation

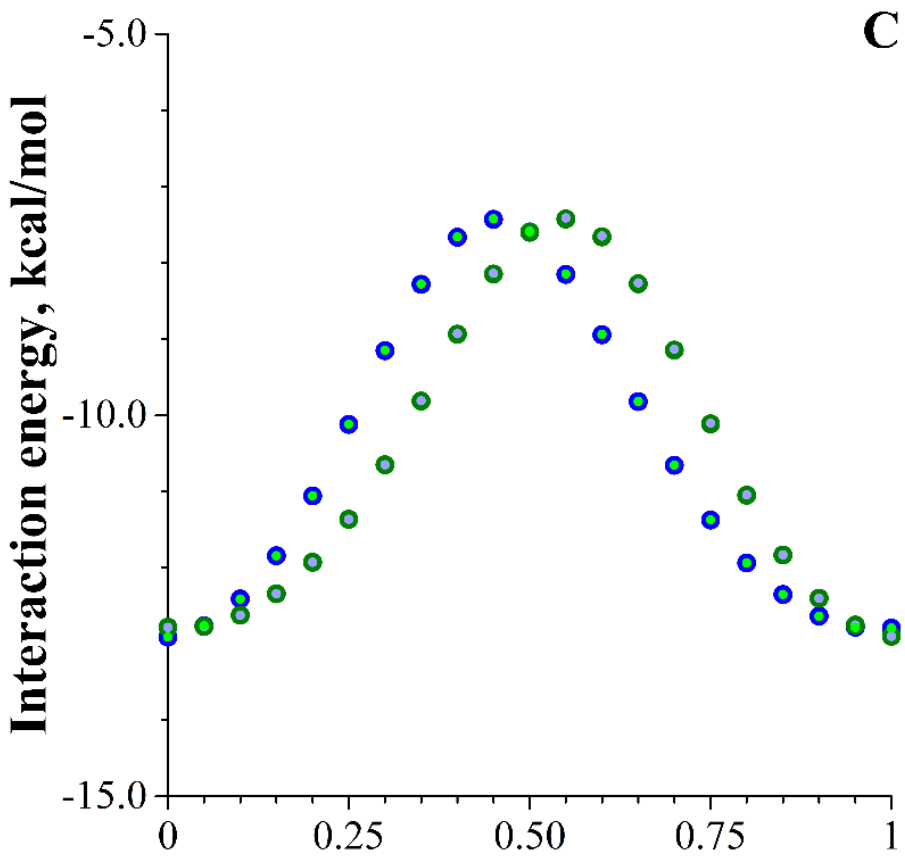

Part of translation

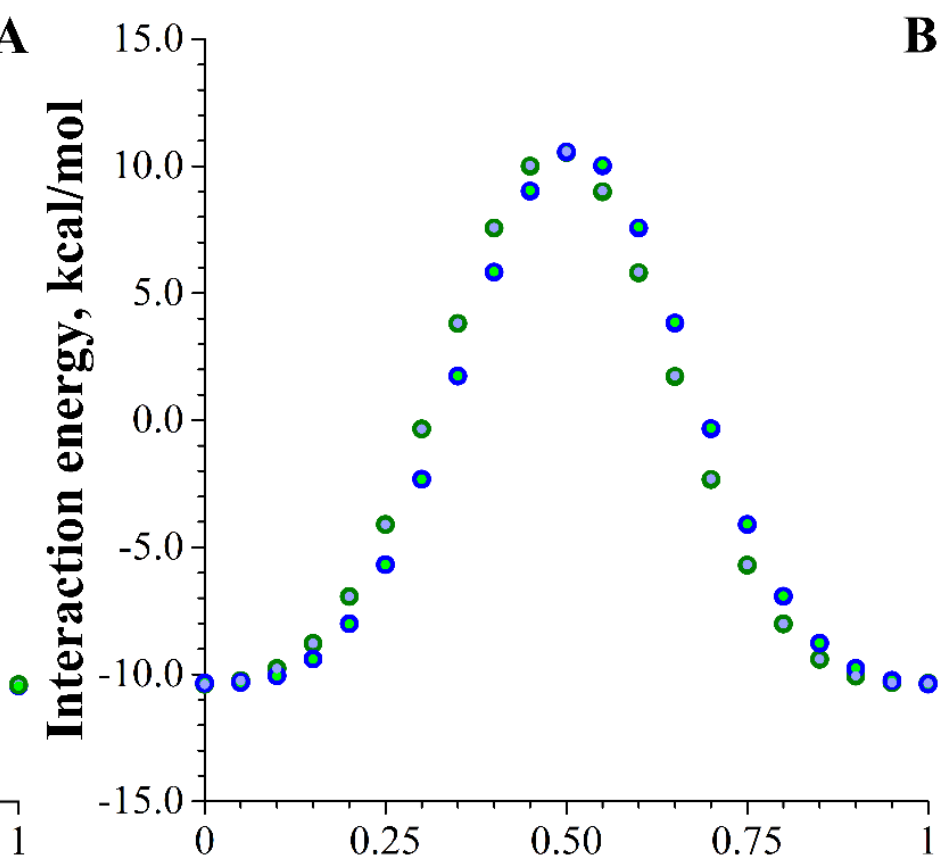

Part of translation

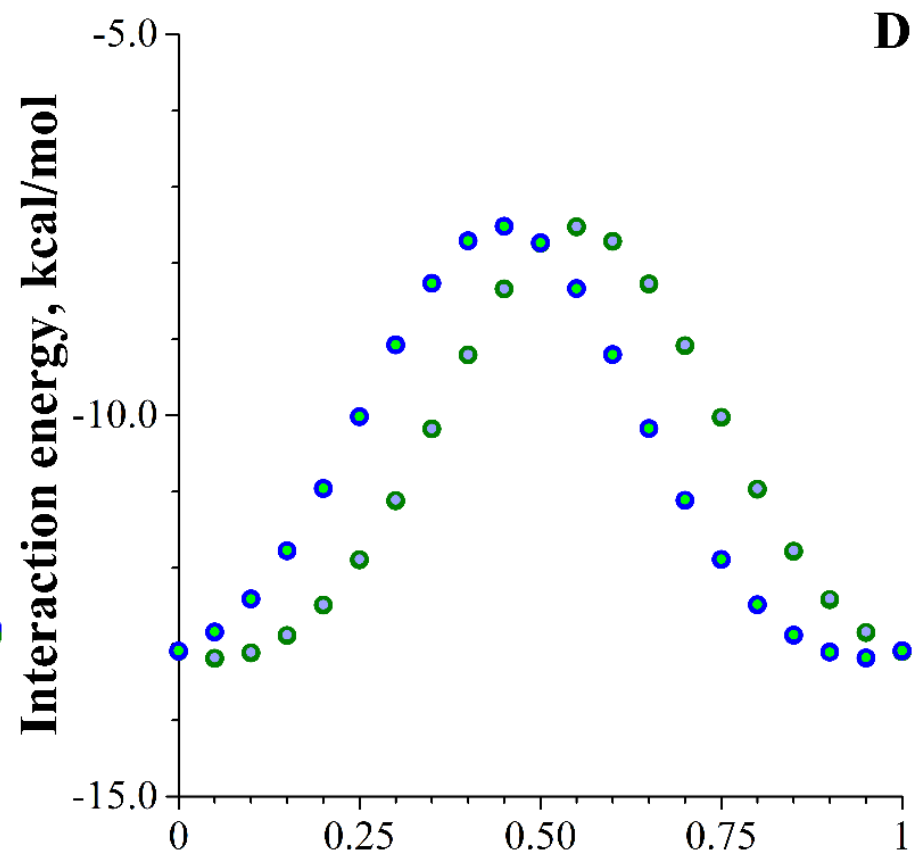

Part of translation

Figure S4. Interaction energy profile for the shifts of different symmetric equivalents of dimeric BUs along the (100) layers in the [010] direction. Blue and pale blue dots correspond to the shifts of the initial dimeric BUs along the $\mathrm{BSM}_{2}$ with higher (big) or lower (small) coordinate by the [100] direction respectively, olive and green dots are for the shifts of the dimeric BUs rotated by $2{ }_{1}$-screw axes once belonging to the polymorphic forms: I (A); II (B); IV, AA-BU as a basic element (C); IV, BB-BU as a basic element (D) 


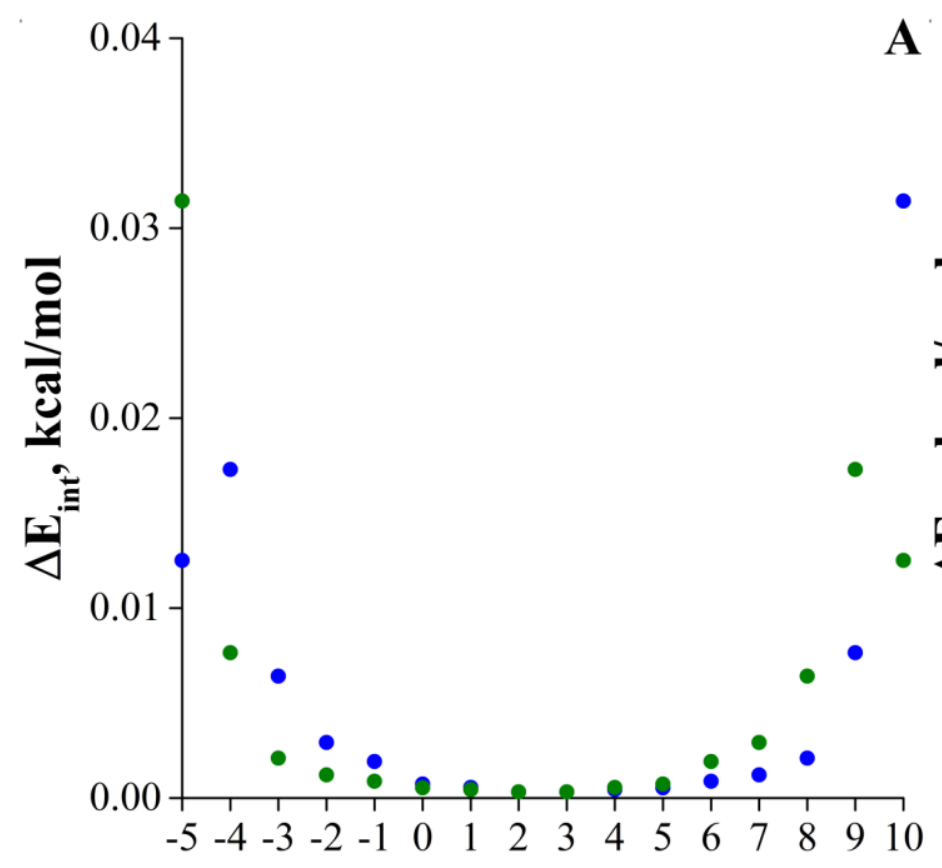

Translation number

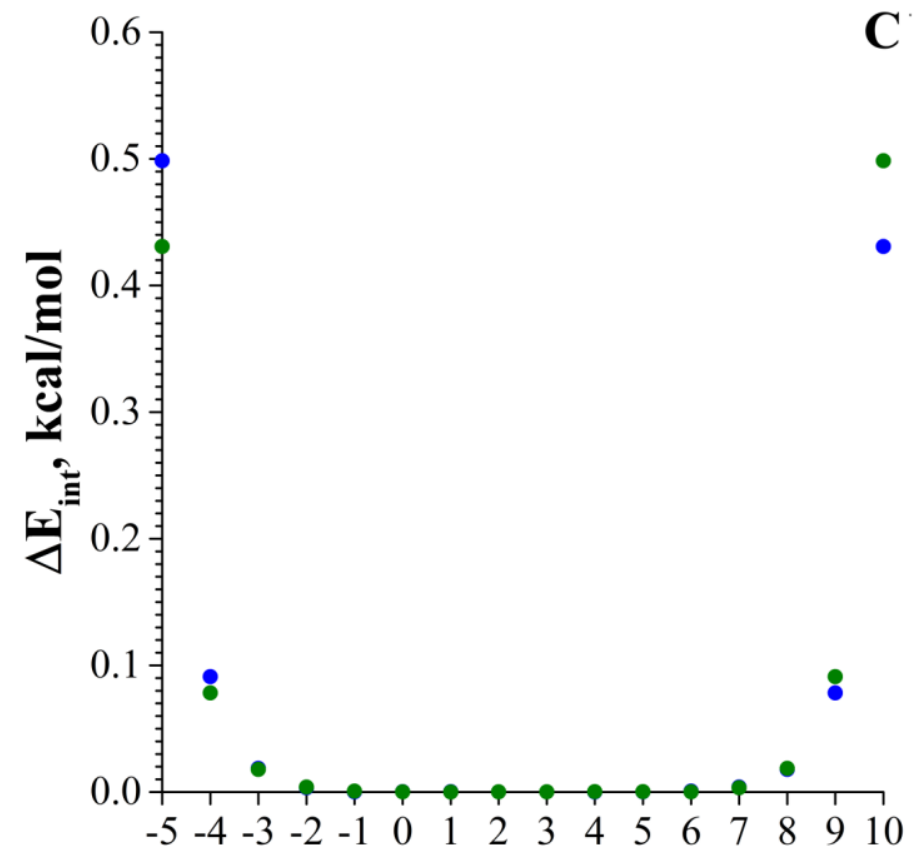

Translation number $\begin{array}{lll}\text { A } & 0.04 & \text { B }\end{array}$

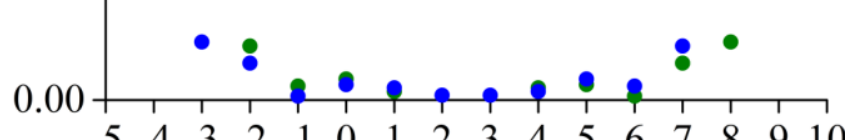

Translation number

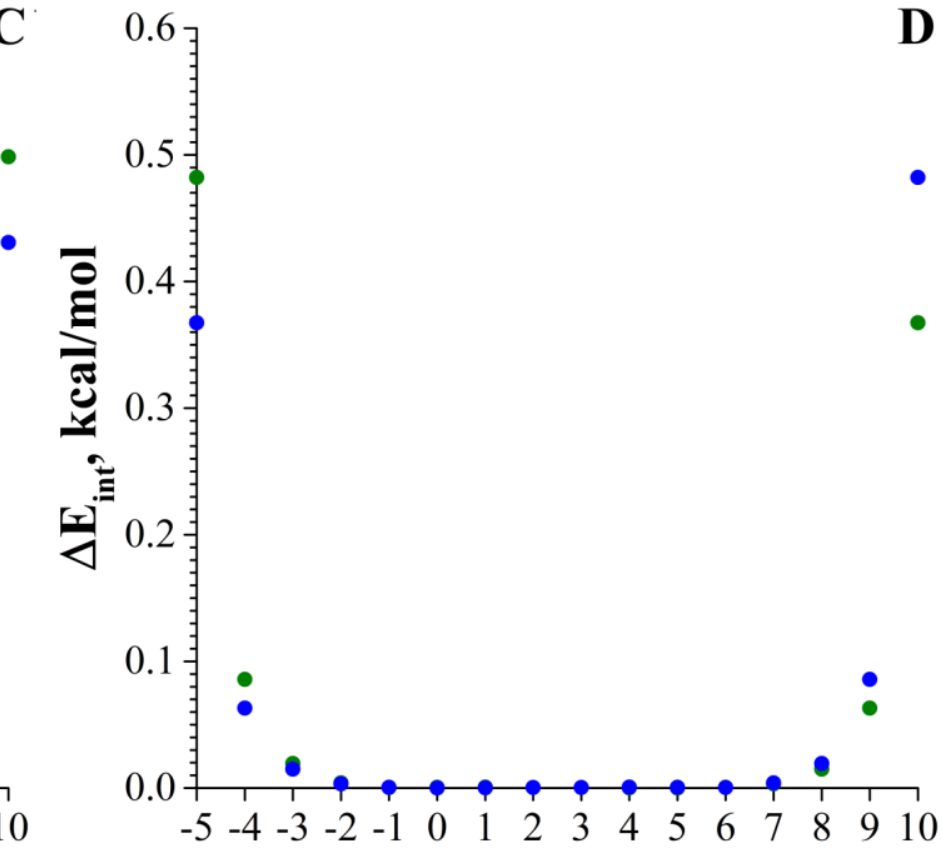

Translation number

Figure S5. Interaction energy deviations occurring between the symmetrically equivalent positions of the dimeric BU as a mobile part (its initial disposition is in blue, disposition rotated by 2 -screw axes once is in olive) during the transitions from the edge to the center beside the neighboring (100) layer between the -5 th and 10th positions in the [010] direction for the polymorphic forms: I (A); II (B); IV, AA-BU as a basic element (C); IV, BB-BU as a basic element (D) 

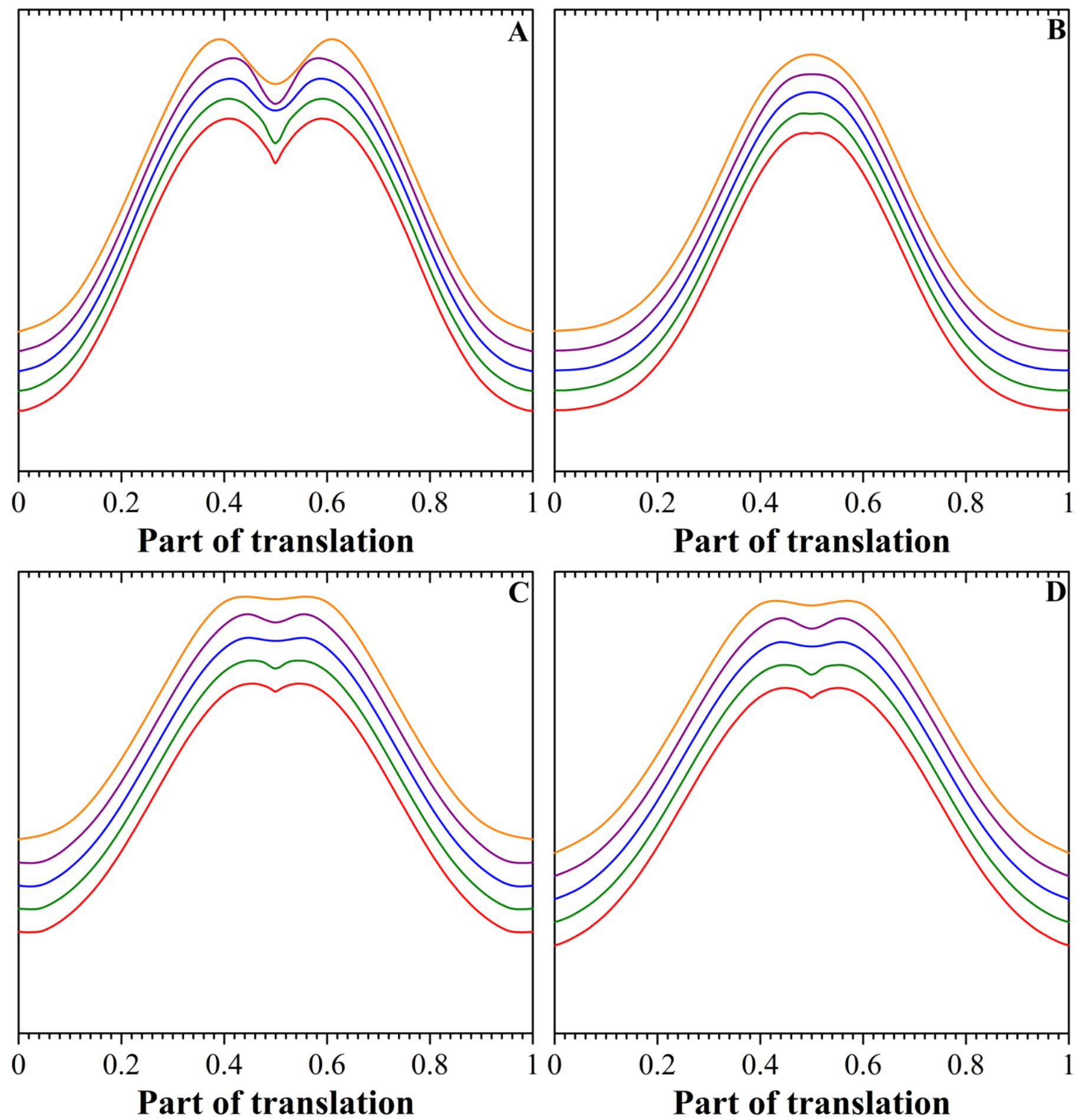

Figure S6. Profile of the interaction energy between the dimeric mobile part and the neighboring (100) layer during the translation between the equivalent positions in the [010] direction with the step size of 1/100 (red), 1/50 (olive), 1/25 (blue), 1/20 (purple), 1/10 (orange) of the corresponding distance for the polymorphic forms: I (A); II (B); IV, AA-BU as a basic element (C); IV, BB-BU as a basic element (D) 


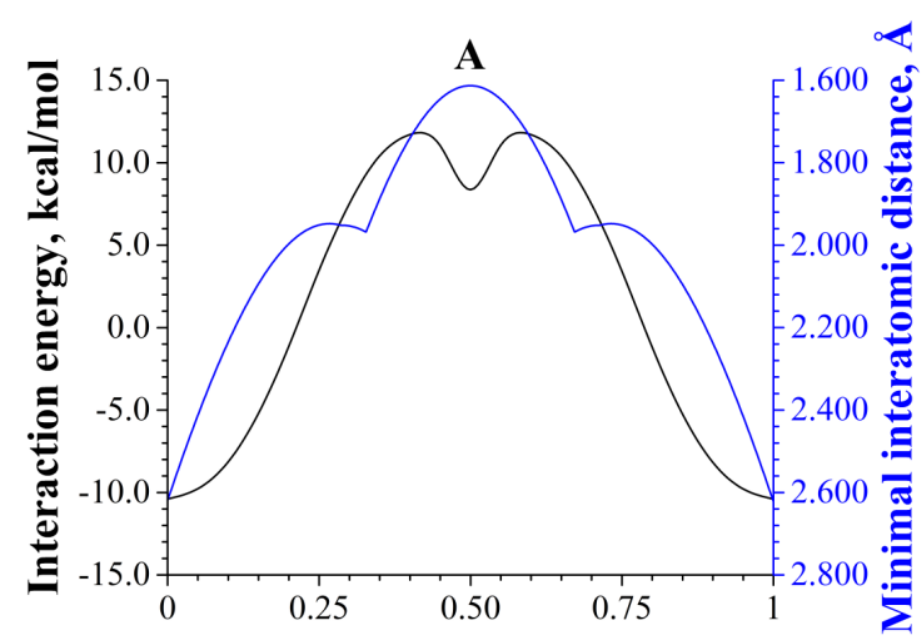

Part of translation

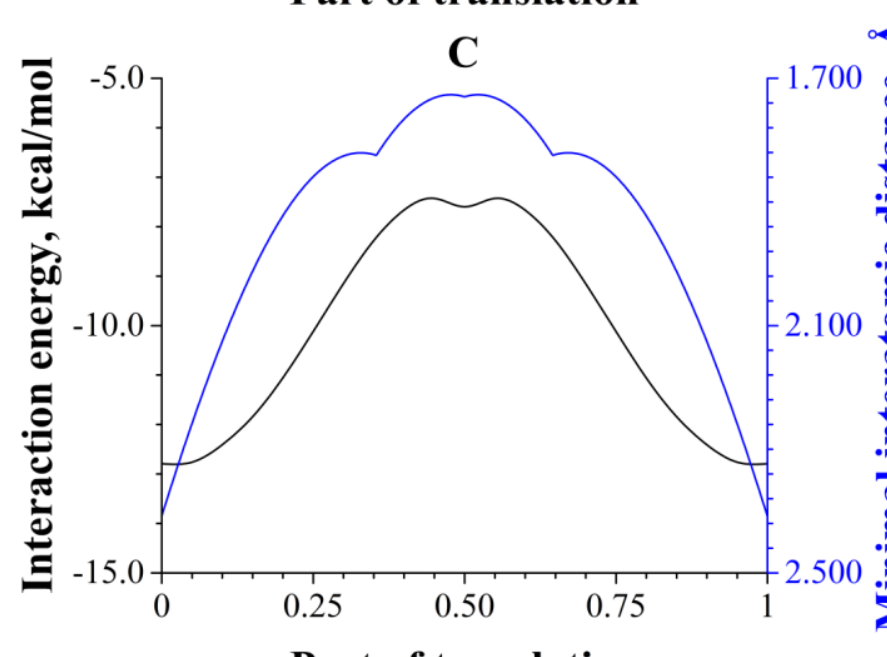

Part of translation

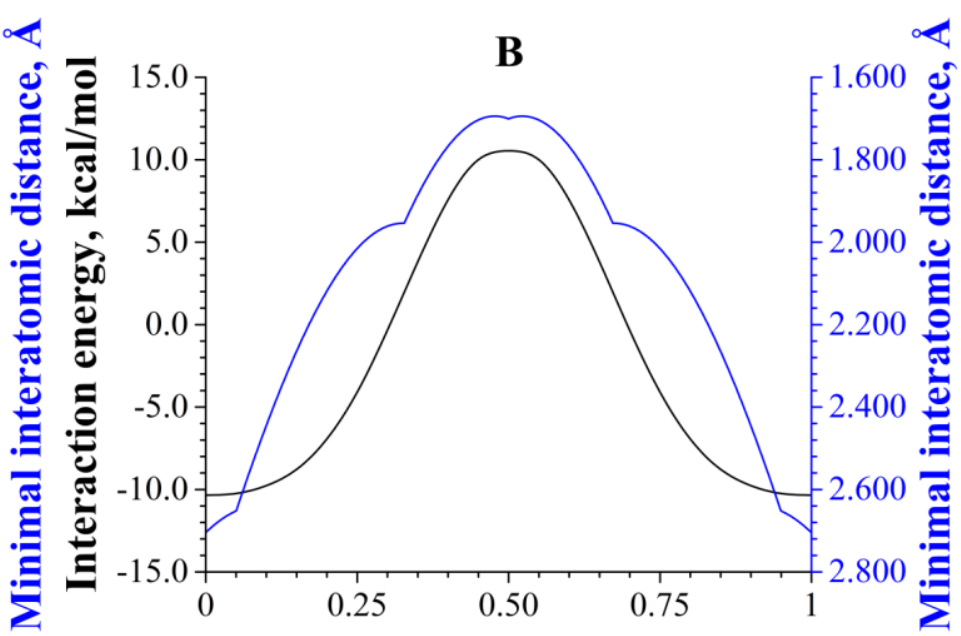

Part of translation

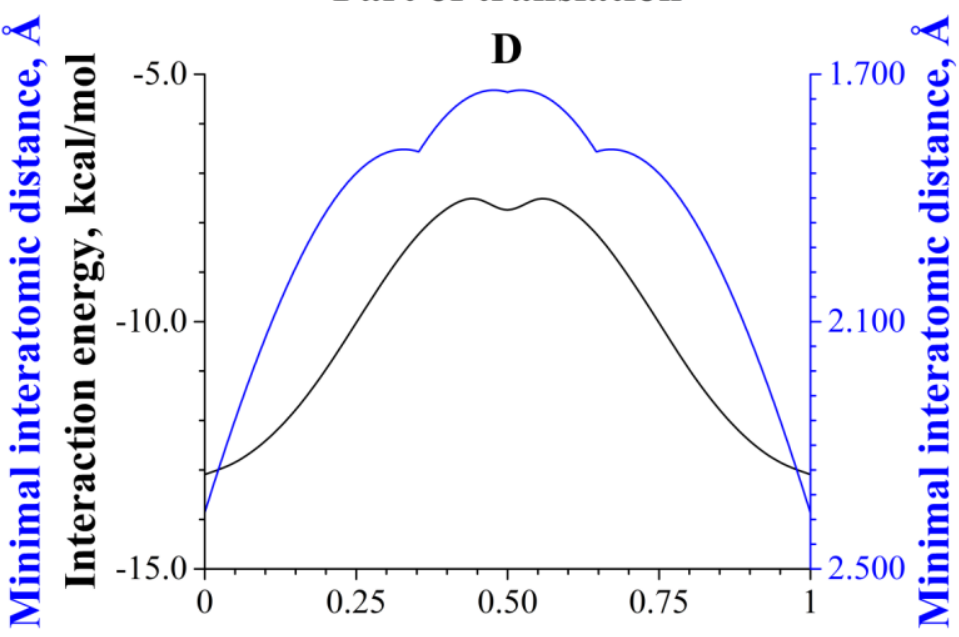

Part of translation

Figure S7. Profiles of the interaction energy (black) and minimal interatomic distance (blue) between the dimeric mobile part and the neighboring (100) layer in the [010] direction for the polymorphic forms: I (A); II (B); IV, AA-BU as a basic element (C); IV, BB-BU as a basic element (D) 

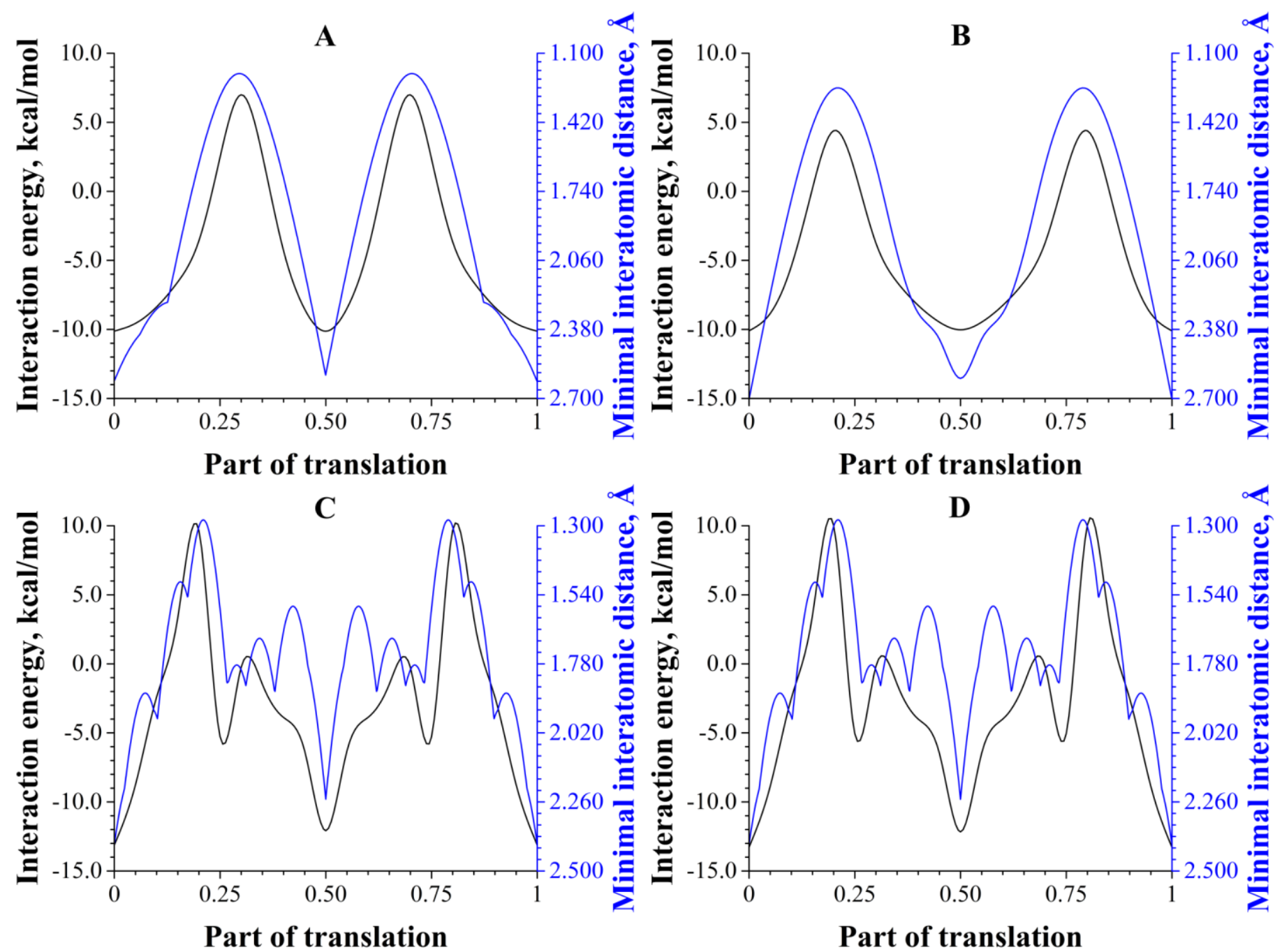

Figure S8. Profiles of the interaction energy (black) and minimal interatomic distance (blue) between the dimeric mobile part and the neighboring (100) layer in the [001] direction for the polymorphic forms: I (A); II (B); IV, AA-BU as a basic element (C); IV, BB-BU as a basic element (D) 


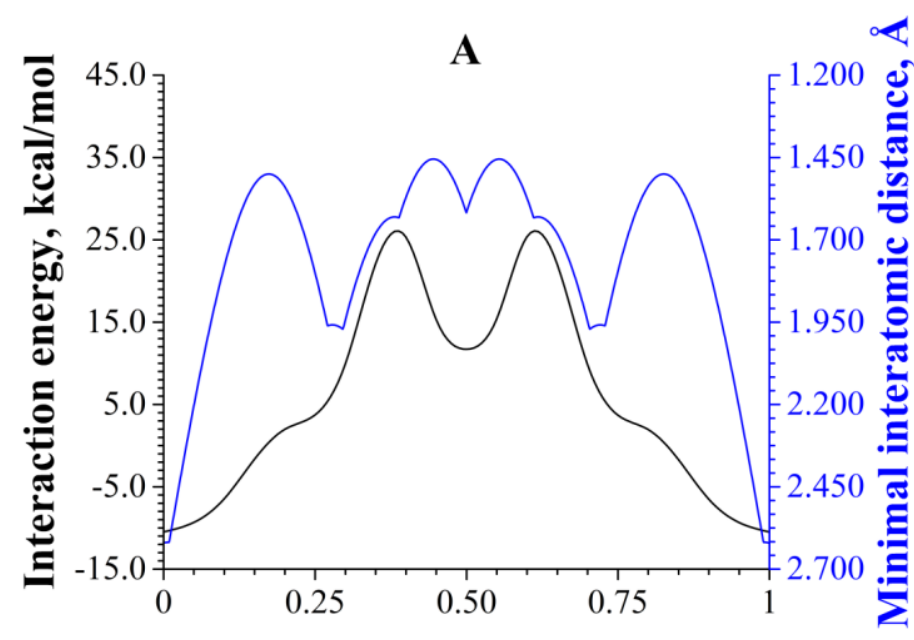

Part of translation

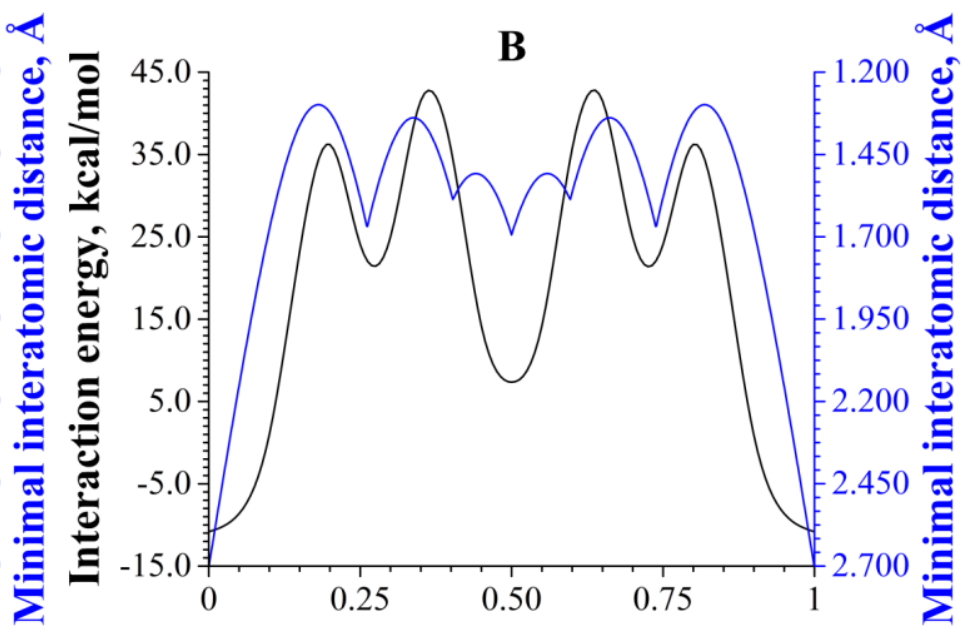

Part of translation

Figure S9. Profiles of the interaction energy (black) and minimal interatomic distance (blue) between the dimeric mobile part and the neighboring (100) layer in the [011] direction for the polymorphic forms $\mathbf{I}(\mathrm{A})$ and II (B)

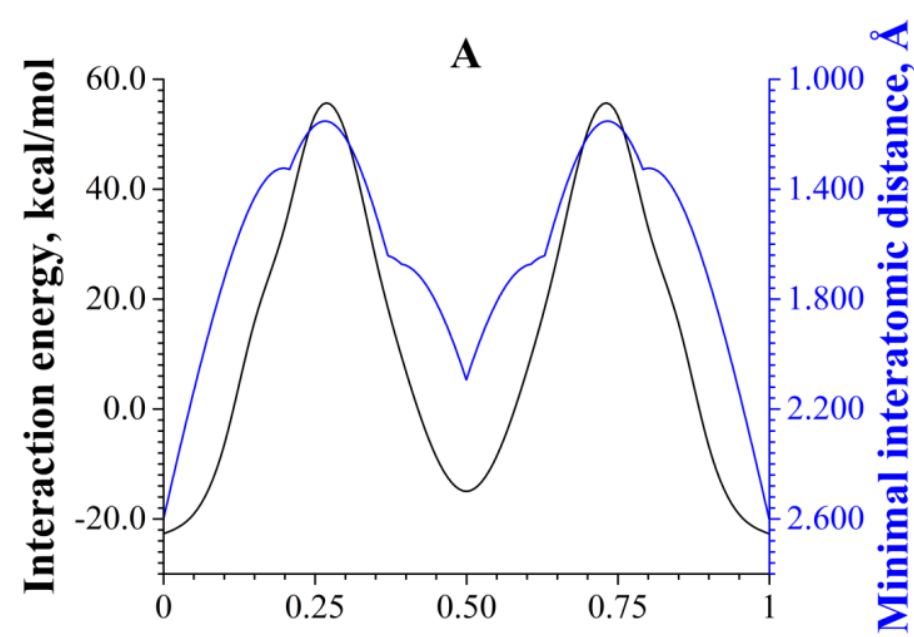

Part of translation

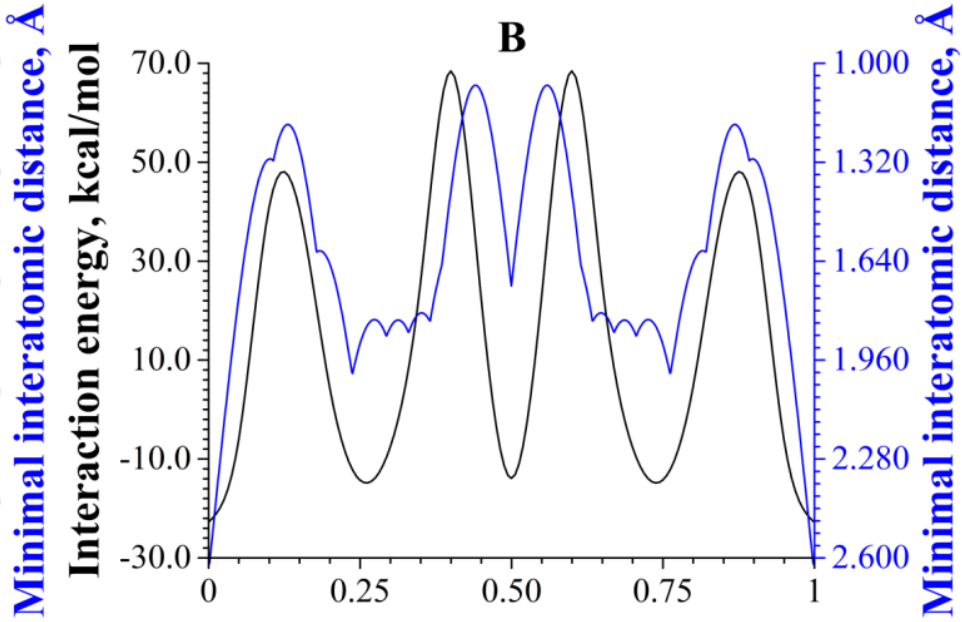

Part of translation

Figure S10. Profiles of the interaction energy (black) and minimal interatomic distance (blue) between the dimeric mobile part and the neighboring (001) layer in the [100] direction for the polymorphic form I (A) and the neighboring (-102) layer in the [201] direction for the polymorphic form II (B) respectively 


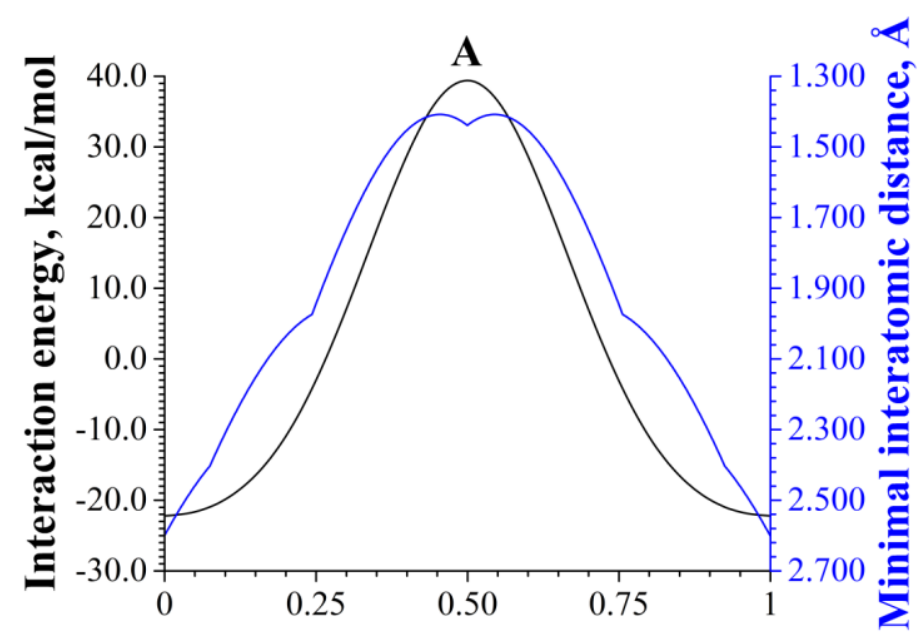

Part of translation

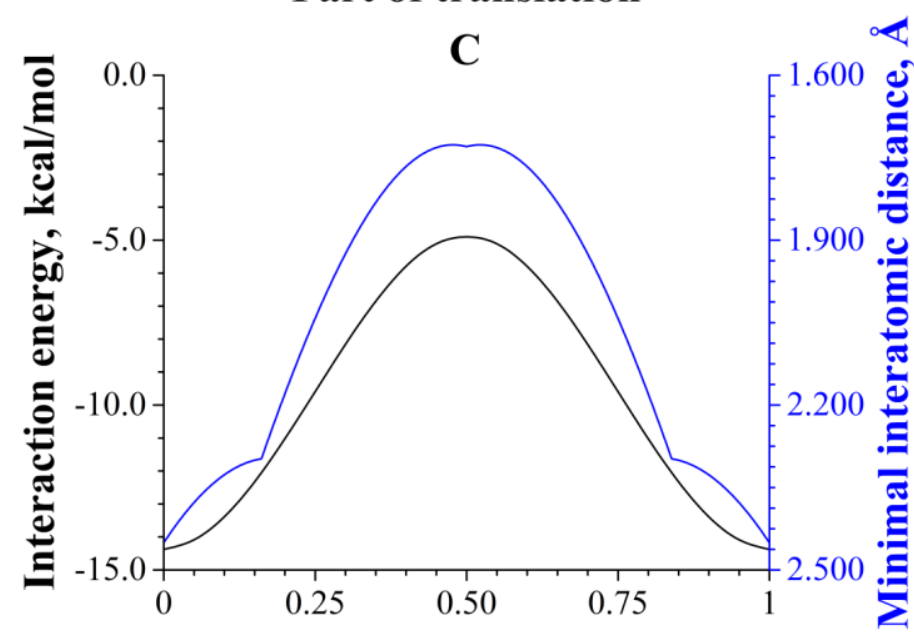

Part of translation

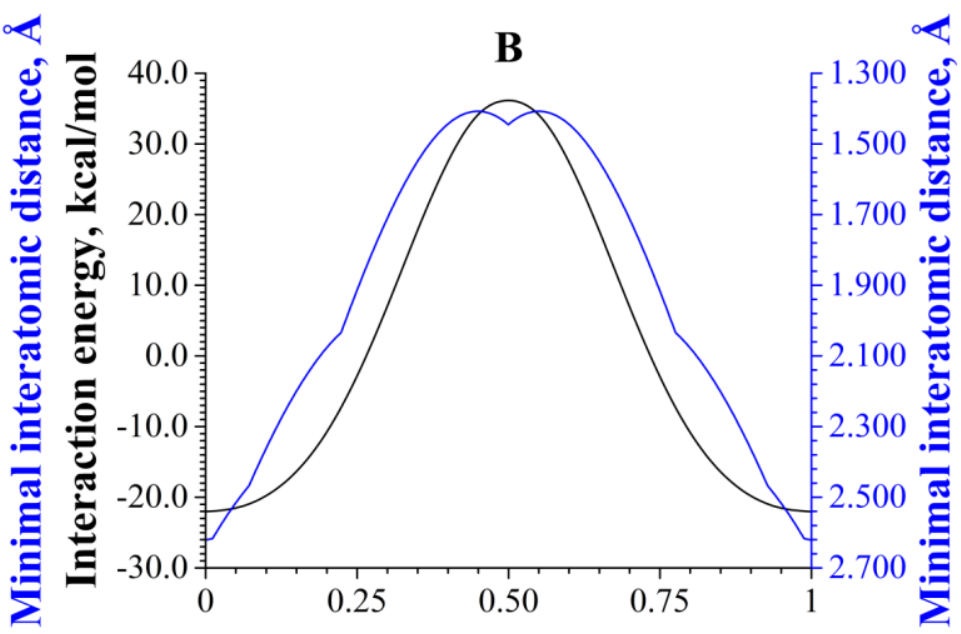

Part of translation

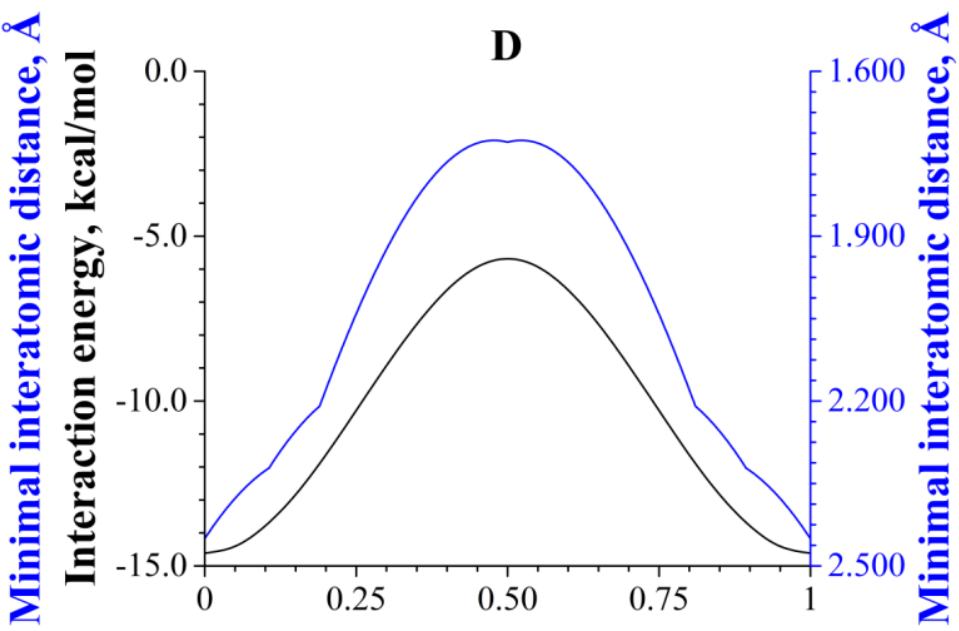

Part of translation

Figure S11. Profiles of the interaction energy (black) and minimal interatomic distance (blue) between the dimeric mobile part and the neighboring (001) layers for the polymorphic forms I (A); IV, AA-BU as a basic element (C); IV, BB-BU as a basic element (D) and the neighboring (-102) layer for the polymorphic form II (B) in the [010] direction respectively 


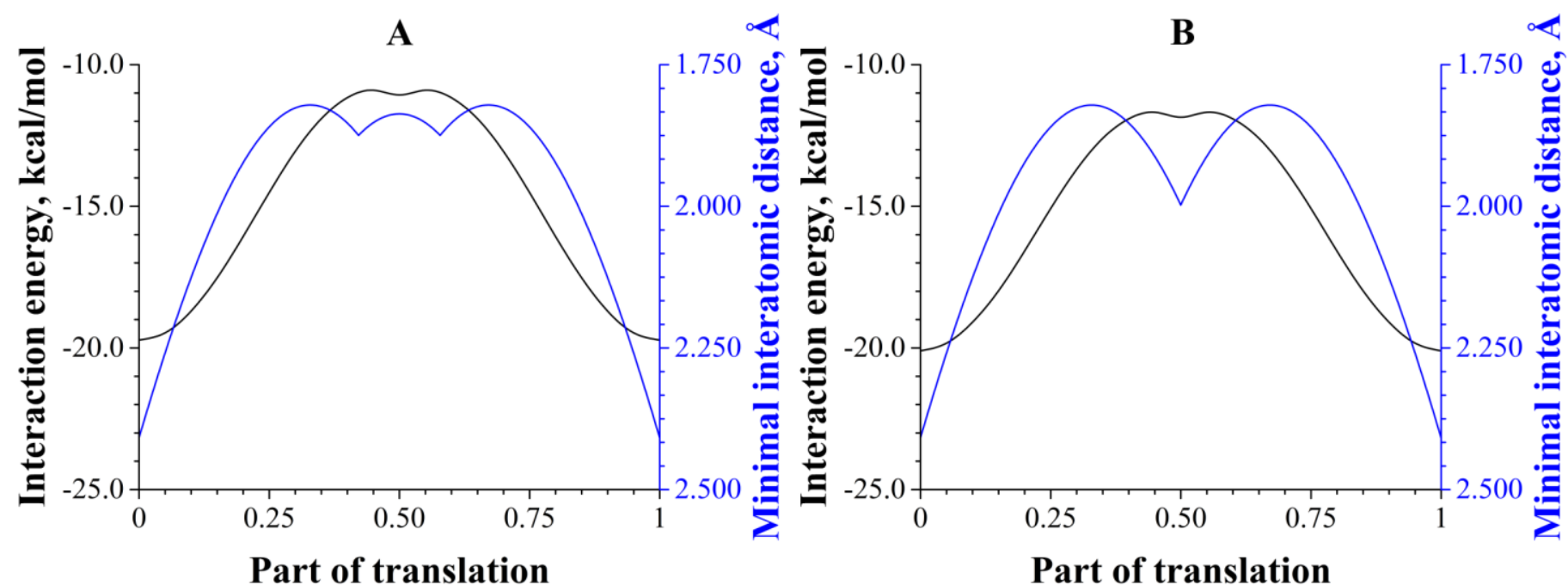

Figure S12. Profiles of the interaction energy (black) and minimal interatomic distance (blue) between the dimeric mobile part and the neighboring (-101) layer in the [010] direction for the polymorphic form IV, AA-BU as a basic element (A) and BB-BU as a basic element (B) 